\title{
Research Paper \\ A Comparative Study on the Burden of Disease of Schizophrenia, Bipolar Dis- order Type I, and Autism Spectrum Disorder on the Family Caregivers in Iran
}

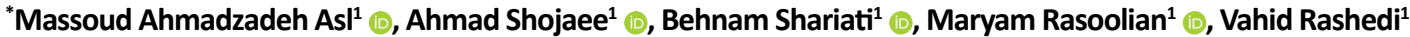

1. Tehran Psychiatric Institute, Iran University of Medical Sciences, Tehran, Iran

Critation Ahmadzadeh Asl M, Shojaee A, Shariati B, Rasoolian M, Rashedi V. [A Comparative Study on the Burden of Disease of Schizophrenia, Bipolar Disorder Type I, and Autism Spectrum Disorder on the Family Caregivers in Iran (Persian)]. Archives of Rehabilitation. 2021; 22(3):320-341. https://doi.org/10.32598/RJ.22.3.3143.1

https://doi.org/10.32598/RJ.22.3.3143.1

Received: 20 Dec 2019 Accepted: 22 Feb 202 Available Online: 01 Oct 202

Keywords:

Schizophrenia, Autism spectrum disorder, Bipolar disorder, Burden of disease

\section{A B STRACT}

Objective Patients with severe psychiatric diseases, due to the debilitating and chronic nature of these diseases, requires prolonged care by family and other rated people. In addition to the patient, these diseases affect the caregiver and create high psychological, social, and individual pressure to take care of themselves. This study aims to compare the burden of schizophrenia, Bipolar Disorder (BD) type 1, and Autism Spectrum Disorder (ASD) on the family caregivers in Iran.

Materials \& Methods In this descriptive-analytical study, using the non-probability sampling method, 450 family caregivers of patients with schizophrenia, BD type 1 , and ASD were selected based on the inclusion criteria. Data collection tools comprised a demographic checklist, short-form Zarit Burden Interview (ZBI-12), and the Depression, Anxiety, and Stress Scale (DASS). The questionnaires were distributed to the patients selected from the Psychiatric Institute of Tehran, Iran Psychiatric Hospital, and Ali Asghar Hospital. The collected data were analyzed using descriptive statistics, ANOVA for evaluating the relationship of demographic factors with the amount and severity of disease burden, and interclass correlation coefficient in SPSS v. 22

Results The disease burden was higher on caregivers of ASD patients, followed by that of BD type 1 and schizophrenia patients. The highest and lowest hours of care were related to the ASD and schizophrenia groups, respectively. Women made up the majority of family caregivers. The educational level of family caregivers was higher in the BD type 1 group and was lower in the schizophrenia group. Most caregivers in the BD type 1 group were employed, while most of them in the schizophrenia group were housewives. The lowest and highest income levels were related to the family caregivers of ASD and schizophrenia groups, respectively. The highest and lowest hospitalization frequencies were seen in the BD type 1 and ASD groups, respectively. Conclusion The burden of three diseases on the family caregivers is high. It is recommended that staterun consulting and screening centers be more active in this field. Because of the low-income level of some family caregivers, it is better to plan for more employment of family caregivers with the assistance of governmental and non-governmental organizations. It is better to hold strategic classes for the family caregivers to reduce their disease burden. Different methods to reduce the burden of diseases in caregivers, such as lowering care hours and using respite care and respite recess and dividing tasks between caregivers, using social or daycare services, can reduce their symptoms of depression and anxiety. Their depression and anxiety should be monitored, and pharmacological and non-pharmacological measures should be used for their treatment.

\section{Corresponding Author:}

Massoud Ahmadzadeh Asl, PhD.

Address: Tehran Psychiatric Institute, Iran University of Medical Sciences, Tehran, Iran.

Tel: +98 (912) 4869713

E-Mail: ahmadshojah28@gmail.com 


\section{English Version}

\section{Introduction}

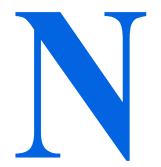

on-communicable diseases are responsible for the deaths of more than 35 million people each year [1]. Policymaking and managing the healthcare programs should know the burden of disease as the most objective evidence [2]. Schizophrenia, Bipolar Disorder (BD), and Autism Spectrum Disorder (ASD) have common genetic etiologies [3]. In developing countries such as Iran, the families of schizophrenia patients are involved in the longterm rehabilitation process [4]. Schizophrenia is a mental disorder characterized by distortions in thinking, emotion, behaviors, and perception of reality [5]. Schizophrenia is one of the most severe neuropsychiatric disorders [6]. According to the World Health Organization, schizophrenia is the eighth leading cause of disability-adjusted life year in the age group of 15-44 years worldwide [7]. In pathological systems, schizophrenia is mainly known as a set of signs and symptoms such as delusions, hallucinations, and disorganized speech and behavior [8]. One study showed a significant relationship between quality of life and coping strategies [9]. Patients with schizophrenia have low levels of education, income, and social status [10]. BD or manic-depressive illness, is associated with acute seizures $[11,12]$. It is difficult to estimate the annual incidence of $\mathrm{BD}$ [11] accurately, and it appears that $20 \%$ of patients with clinical signs of depression are bipolar [12]. BD is a brain disorder that causes severe mood swings and changes in body energy levels [13, 14]. Drug adherence is low among patients with BD [15]. The performance of patients with schizophrenia and $\mathrm{BD}$, as measured by the Wisconsin test, is poorer than the healthy population [16].

One of the most severe developmental disorders is ASD that occurs in early childhood (usually before the age of 3 ) [17]. A study showed that quality of life therapy increases the psychological wellbeing of mothers of children with ASD [18]. Another study showed that mindfulness-based treatment improves the quality of life and coping styles of mothers of children with ASD [19]. According to Zhang et al., the families of patients with BD undergo a lot of stress during the acute treatment and recovery period [20]. According to Matson et al., the variety and intensity of care roles may lead to psychological problems in family caregivers [21]. According to Ivey, the psychological burden of caring for a mentally ill person can reduce the quality of provided care [22]. The most critical causes of recurrence of mental disorders are discontinuation or irregular use of medication and disruption in the relationship between the patient and the family [23]. In most previous studies, the sample size is small, and the disease burden in the families of patients with depression and BD was generally measured and compared with each other. In most studies, the families of patients with schizophrenia and ASD and the relationship of disease burden with social and economic status and income have not been studied. The research and comparison of schizophrenia, BD, and ASD are necessary since they have a high disease burden, and all are disabling and chronic. Given the genetic similarities and overlap of symptoms in these three diseases, we aimed to compare the burden of these diseases using a larger sample size. In this study, we tried to answer how much the burden of these diseases is and what factors are related to the burden of these diseases.

\section{Materials and Methods}

This descriptive study was conducted on 450 patients (150 with schizophrenia, 150 with $\mathrm{BD}$, and 150 with ASD). The sample size was determined using Cochran's formula. The inclusion criteria were being 18 years or older, having at least a primary education, having a family member with one of the three diseases, having been diagnosed by a physician on an outpatient or inpatient basis, having the disease in the past year, and being under treatment, not being hospitalized in the past month (because the study outcome can be affected due to the reported high burden of disease in families). The exclusion criteria were unwillingness to continue study participation and having a cognitive disorder or any condition that can impede them from answering the questions correctly (e.g. Alzheimer disease). A sampling of patients with schizophrenia and BD was performed in Iran Psychiatric Hospital, Tehran Psychiatric Institute, and Rasoul-e Akram Hospital. Also, sampling of ASD patients was performed in Ali Asghar Hospital, Tehran Psychiatric Institute, and the Center for the Treatment of Autistic Disorders. From each center, 17 families were selected.

The short form of Zarit Burden Interview (ZBI-12) was used in this study. This questionnaire has 12 items measuring the burden of care on caregivers of patients rated on a Likert scale from 0 to 4 and has a total score of 0-48. Its acceptable validity and reliability have been shown in various studies [24]. For its Persian version, Navidian et al. reported a good validity based on the opinions of a panel of experts and test-retest reliability of 0.94 [25]. The Depression, Anxiety, and Stress Scale (DASS) were also used in this study to measure anxiety and depression in the family of patients with mental disorders. One copy of the questionnaires along with a demographic checklist (surveying age, gender, relationship with the patient, level of education, employment status, income level, medical history, etc.) was provided to the one accompanying the patient (spouse, parents, child over 18 , sibling or other person living with the 
patient). Then, two more copies of the questionnaires and the checklist were given to the patient and asked him or her to be given to two other family members/partners. The obtained data were analyzed in SPSS v. 22.

\section{Results}

According to Table 1, the Mean \pm SD age of the caregivers was $43.27 \pm 0.70$ years, and there was a significant difference between the three groups of caregivers in terms of age $(\mathrm{P}=0.001)$. The highest hours of patient care were related to the caregivers of ASD patients, but there was no significant difference between the three groups $(\mathrm{P}=0.023)$. The highest hours of respite care were related to the caregivers of schizophrenia patients, but there was no significant difference between the three groups $(\mathrm{P}=0.034)$.

According to Table 2, the number of female and married caregivers was higher in all three groups. In terms of occupation, most of the caregivers in the schizophrenia group were housewives (28\%), while most caregivers in the BD and ASD groups were employed (32\% and $51.3 \%$, respectively). More than $92 \%$ of caregivers of patients with schizophrenia had no history of mental illness. Most of the caregivers in the three groups had no record of chronic disease. In the schizophrenia group, the highest income level of caregivers was 10-30 million Rials (42\%), while in the $\mathrm{BD}$ group, most of them had income level $<10$ million
Rials (20.7\%). In the ASD group, most caregivers had no income (36.7\%). According to Table 3, the mean burden of the disease reported by caregivers in patients with ASD was 23.54, which was more than those of BD and schizophrenia. The mean burden of BD (19.87) was higher than that of schizophrenia. There was a significant difference in the mean ZBI-12 score between the three groups $(\mathrm{P}<0.05)$. The mean DASS score in the three groups of schizophrenia, BD, and ASD was 18.36, 18.39, and 22.91, respectively.

\section{Discussion and Conclusion}

In this study, the mean burden of the disease reported by caregivers of patients with ASD was 23.54, which was more than those of BD and schizophrenia. The mean burden of illness of BD (19.87) was higher than that of schizophrenia. There was a significant difference between the three groups regarding the mean ZBI-12 score $(\mathrm{P}<0.05)$. The mean DASS scores in the three groups of schizophrenia, $\mathrm{BD}$, and ASD was 18.36, 18.39, and 22.91, respectively. There was a significant difference in the mean DASS score between the three groups $(\mathrm{P}<0.05)$.

The linear correlation coefficient for the mental status of caregivers for the total patients was 0.574 , indicating a moderate correlation between the independent variable and the dependent variable. The effect size $(0.524)$ revealed that independent variables could predict more than $52 \%$ of the

Table 1. Mean values of age, hours of care, and free hours of care in caregivers and patients and the test results

\begin{tabular}{|c|c|c|c|c|c|}
\hline Groups & \multicolumn{2}{|c|}{ Variabless } & Mean $\pm S D$ & $x^{2}$ & $\mathbf{P}$ \\
\hline & & Schizophrenia & $42.22 \pm 1.28$ & & \\
\hline & Age & $\mathrm{BD}$ & $48.41 \pm 1.37$ & 23.74 & $<0.001$ \\
\hline & & ASD & $39.19 \pm 0.8$ & & \\
\hline & & Schizophrenia & $10.63 \pm 0.72$ & & \\
\hline \multirow[t]{6}{*}{ Caregivers } & Hours of care & $\mathrm{BD}$ & $10.88 \pm 0.67$ & 7.57 & 0.023 \\
\hline & & ASD & $12.14 \pm 0.59$ & & \\
\hline & & Schizophrenia & $93.47 \pm 5.10$ & & \\
\hline & $\begin{array}{c}\text { Hours of respite } \\
\text { care }\end{array}$ & $\mathrm{BD}$ & $88.91 \pm 4.71$ & 6.74 & 0.0234 \\
\hline & & ASD & $83.03 \pm 4.14$ & & \\
\hline & & Schizophrenia & $37.62 \pm 1.09$ & & \\
\hline \multirow[t]{2}{*}{ Patients } & Age & $\mathrm{BD}$ & $36.88 \pm 0.83$ & 295.147 & $<0.001$ \\
\hline & & ASD & $9.21 \pm 0.34$ & & \\
\hline
\end{tabular}


Table 2. Percentages of caregivers based on demographic factors in each group and the test results

\begin{tabular}{|c|c|c|c|c|c|c|}
\hline \multirow{2}{*}{\multicolumn{2}{|c|}{ Characteristics }} & \multicolumn{3}{|c|}{$\%$} & \multirow{2}{*}{$x^{2}$} & \multirow{2}{*}{$\mathbf{P}$} \\
\hline & & Schizophrenia & BD & ASD & & \\
\hline \multirow{3}{*}{ Gender } & Male & 46 & 42 & 45.3 & & \\
\hline & & & & & 0.558 & 0.757 \\
\hline & Female & 54 & 58 & 54.7 & & \\
\hline \multirow{5}{*}{ Marital status } & Married & 71.3 & 56 & 90 & & \\
\hline & Single & 23.3 & 3.37 & 6.7 & & \\
\hline & & & & & 45.52 & $<0.001$ \\
\hline & Widow/widower & 3.3 & 3.3 & 2 & & \\
\hline & Divorced & 2 & 3.3 & 1.3 & & \\
\hline \multirow{5}{*}{$\begin{array}{l}\text { Relationship with } \\
\text { the patient }\end{array}$} & Mother & 28 & 20.7 & 44.7 & & \\
\hline & Father & 24 & 16 & 40 & & \\
\hline & Brother/sister & 28 & 36 & 8 & 128.90 & $<0.001$ \\
\hline & Child & 3.9 & 14 & 0 & & \\
\hline & Other & 10.7 & 13.3 & 7.3 & & \\
\hline \multirow{5}{*}{ Educational level } & $\begin{array}{l}\text { Lower than high } \\
\text { school }\end{array}$ & 44.7 & 32.7 & 26 & & \\
\hline & Diploma & 32 & 28.7 & 37.3 & & \\
\hline & $\begin{array}{c}\text { Bachelor's/ } \\
\text { Master's degree }\end{array}$ & 20.7 & 36.7 & 30 & 27.34 & 0.001 \\
\hline & Doctoral degree & 1.3 & 1.3 & 6.7 & & \\
\hline & None & 1.3 & 0.7 & 0 & & \\
\hline \multirow{7}{*}{ Occupation } & Unemployed & 7.3 & 13.3 & 6 & & \\
\hline & Employed & 27.3 & 32 & 50 & & \\
\hline & Retired & 24.7 & 13.3 & 2.7 & & \\
\hline & & & & & 67.90 & $<0.001$ \\
\hline & College student & 9.3 & 10 & 2.7 & & \\
\hline & Housekeeper & 28 & 28 & 35.3 & & \\
\hline & Others & 3.4 & 3.4 & 3.3 & & \\
\hline \multirow{3}{*}{$\begin{array}{l}\text { History of a } \\
\text { mental illness }\end{array}$} & Yes & 4.7 & 10.7 & 8 & & \\
\hline & No & 92.7 & 88.7 & 92 & 8.83 & 0.065 \\
\hline & $\begin{array}{c}\text { I do not want to } \\
\text { express }\end{array}$ & 2.7 & 0.7 & 0 & & \\
\hline \multirow{5}{*}{$\begin{array}{c}\text { History of a } \\
\text { chronic disease }\end{array}$} & Physical & 7.6 & 12 & 14 & & \\
\hline & Mental & 22.7 & 30.7 & 14 & & \\
\hline & Both & 11.3 & 10.7 & 6 & 21.30 & 0.006 \\
\hline & None & 51.3 & 41.3 & 58.7 & & \\
\hline & $\begin{array}{c}\text { I do not want to } \\
\text { express }\end{array}$ & 8 & 5.3 & 7.3 & & \\
\hline
\end{tabular}




\begin{tabular}{|c|c|c|c|c|c|c|}
\hline \multirow{2}{*}{\multicolumn{2}{|c|}{ Characteristics }} & \multicolumn{3}{|c|}{$\%$} & \multirow{2}{*}{$\chi^{2}$} & \multirow{2}{*}{$\mathbf{P}$} \\
\hline & & Schizophrenia & BD & ASD & & \\
\hline \multirow{6}{*}{$\begin{array}{c}\text { Income, Iranian } \\
\text { Rials }\end{array}$} & $<10$ million & 12 & 20.7 & 10 & \multirow{6}{*}{47.85} & \multirow{6}{*}{$<0.00$} \\
\hline & 10-30 million & 42 & 34 & 28 & & \\
\hline & 30-50 million & 2 & 8 & 14 & & \\
\hline & $>50$ million & 3.3 & 1.3 & 6 & & \\
\hline & No income & 32.7 & 20.4 & 36.7 & & \\
\hline & $\begin{array}{l}\text { I do not want to } \\
\text { express }\end{array}$ & 8 & 15.3 & 5.3 & & \\
\hline \multirow{7}{*}{ Dependents } & 1 & 1.6 & 6.8 & 1.5 & \multirow{7}{*}{25.43} & \multirow{7}{*}{0.114} \\
\hline & 2 & 34.4 & 25 & 31.3 & & \\
\hline & 3 & 34.4 & 45.5 & 50.7 & & \\
\hline & & & & & & \\
\hline & 4 & 18 & 18.2 & 13.4 & & \\
\hline & $>4$ & 9.8 & 4.5 & 1.5 & & \\
\hline & $\begin{array}{l}\text { I do not want to } \\
\text { express }\end{array}$ & 1.6 & 0 & 1.5 & & \\
\hline \multirow{4}{*}{$\begin{array}{l}\text { Number of } \\
\text { patient's } \\
\text { caregivers }\end{array}$} & 2 & 44 & 32.7 & 76 & \multirow{4}{*}{71.16} & \multirow{4}{*}{$<0.00$} \\
\hline & 3 & 50.7 & 54.7 & 16 & & \\
\hline & 4 & 5.3 & 8.7 & 4.7 & & \\
\hline & $>4$ & 0 & 4 & 3.3 & & \\
\hline
\end{tabular}

Abbreviations: BD, Bipolar Disorder; ASD, Autism Spectrum Disorder.

Archivesof
Rehabilitation

changes in the dependent variable, and the remaining $48 \%$ is explained and predicted by variables that were not included in the regression model. This model had a good fit for explaining the dependent variable $(\mathrm{P}=0.0001)$.

The linear correlation coefficient for the mental status of schizophrenia patients' caregivers was 0.566 , indicating a moderate correlation between the independent variable and the dependent variable. The effect size $(0.320)$ revealed that independent variables could predict $32 \%$ of the changes in the dependent variable, and the remaining $78 \%$ is explained and predicted by variables that were not included in the regression model. This model had a good fit for explaining the dependent variable $(\mathrm{P}=0.0001)$. The results regarding the burden of three diseases are consistent with the findings of Navidian (2010) [34].

The linear correlation coefficient for the mental status of BD patients' caregivers was 0.474 , indicating a moderate correlation between the independent variable and the dependent variable. The effect size $(0.422)$ revealed that independent variables could predict $42 \%$ of the changes in the dependent variable, and the remaining 58\% is explained and predicted by variables that were not included in the regression model. This model had a good fit for explaining the dependent variable $(\mathrm{P}=0.0001)$.

The results of this study regarding the financial burden are consistent with the results of Shamsaee et al. (2010) conducted on the needs of family caregivers of bipolar patients. They showed that a large percentage of bipolar patients' family caregivers have financial needs [35]. In a study conducted on patients with schizophrenia admitted to Imam Reza Hospital in Bojnourd, Iran, the disease burden was higher in female caregivers than in male caregivers [36], which is consistent with our results. In a comparative study of mental disorder burden in the family caregivers of patients with depressive disorder, BD, and schizophrenia by Sadeghi et al. [37], the stigma of the disease among the family caregivers of bipolar patients was higher than those of schizophrenia patients, which is consistent with our results.

The linear correlation coefficient for the mental status of ASD patients' caregivers was 0.504 , indicating a moder- 
Table 3. Mean of ZBI-12 and DASS scores in the study groups and the test results

\begin{tabular}{|c|c|c|c|c|}
\hline Variable & Group & Mean \pm SD & $x^{2}$ & P-Value \\
\hline \multirow{3}{*}{ ZBI-12 } & Schizophrenia & $19.08 \pm 13.86$ & \multirow{3}{*}{12.44} & \multirow{3}{*}{0.002} \\
\hline & $\mathrm{BD}$ & $19.78 \pm 12.79$ & & \\
\hline & ASD & $23.54 \pm 11.02$ & & \\
\hline \multirow{3}{*}{ DASS } & Schizophrenia & $18.36 \pm 14.22$ & \multirow{3}{*}{9.83} & \multirow{3}{*}{0.007} \\
\hline & $\mathrm{BD}$ & $18.39 \pm 13.87$ & & \\
\hline & ASD & $22.91 \pm 15.00$ & & \\
\hline
\end{tabular}

BD: Bipolar Disorder; ASD: Autism Spectrum Disorder; ZBI-12: Zarit Burden Interview; DASS: the Depression, Anxiety, and Stress Scale.

ate correlation between the independent variable and the dependent variable. The effect size $(0.254)$ revealed that independent variables could predict more than $25 \%$ of the changes in the dependent variable, and the remaining $75 \%$ is explained and predicted by variables that were not included in the regression model. This model had a good fit for explaining the dependent variable $(\mathrm{P}=0.0001)$.

This study had some limitations such as lack of literature in Persian about the subject, lack of cooperation of some caregivers in answering some questions, intentional mistakes in answering due to poor psychological conditions and fatigue in a small number of caregivers, and getting help from others to answer some questions. The governmental counseling centers should hold strategic classes for family caregivers to reduce the burden of diseases because they belong to low-income families. Moreover, when obtaining information, the researcher should pay more attention to the mental condition and readiness of caregivers. Different methods to reduce the burden of diseases in caregivers, such as lowering care hours and using respite care and respite recess and dividing tasks between caregivers, using social or daycare services, can reduce the symptoms of depression and anxiety in them. Caregivers' mental health monitoring is also recommended as part of the patient's care package, and pharmacological and non-pharmacological measures should be used to treat their depression and anxiety.

Given that women constitute the majority of caregivers, long-term planning using educational interventions should be done for their needs and concerns. In future studies, it is recommended to investigate the facilities available in the houses of caregivers (e.g. the number of rooms) and assess their special care abilities and talents to use these facilities and capabilities to reduce the burden of these diseases. Furthermore, the relationship of interior design, decoration, and arrangement of furniture in caregivers' houses, the number of building units, neighborhoods, cities and villages, and different climates, as well as the job performance of caregivers with the burden of three diseases should be assessed. It is recommended that further studies be conducted using online questionnaires anonymously, and the results are compared with those studies where questionnaires were completed in person.

\section{Ethical Considerations}

Compliance with ethical guidelines

All ethical principles are considered in this article. The participants were informed about the purpose of the research and its implementation stages. They were also assured about the confidentiality of their information. They were free to leave the study whenever they wished, and if desired, the research results would be available to them.

Funding

This research did not receive any grant from funding agencies in the public, commercial, or non-profit sectors.

\section{Authors' contributions}

All authors equally contributed to preparing this article.

\section{Conflict of interest}

The authors declared no conflict of interest. 


\section{Acknowledgments}

We want to thank the esteemed professors of the Faculty of Psychiatry of Iran University of Medical Sciences and the staff and patients of Iran Psychiatric Hospitals and Rasoul Akram (PBUH) and Ali Asghar (AS) and the staff of the Tehran Psychiatric Institute and the management and students of Ain Mehrvarzi School. 
مقايسه بار بيمارى خانواده افراد مبتلا به اسكيزوفرنيا، دوقطبى نوع يك و اختالالات طيف اوتيسم

\author{
"مسعود احمدزاده اصل' (-)، احمد شجاعى' (-) بهنام شريعتى' (-)، مريم رسوليان' (-) وحيد راشدى'
}

ا ا انستيتو روانيزشكى تهران، دانشكاه علوم يزشكى ايران، تهران، ايران.

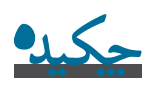

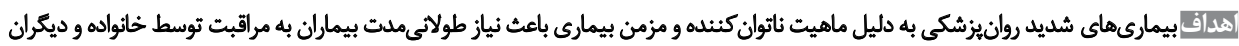

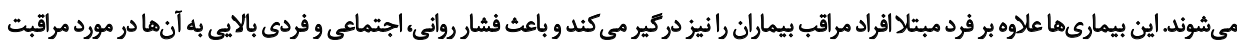

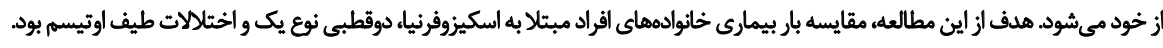

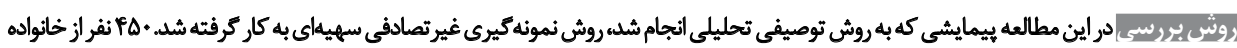

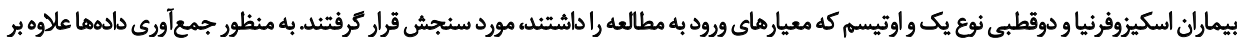

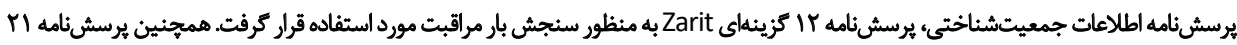

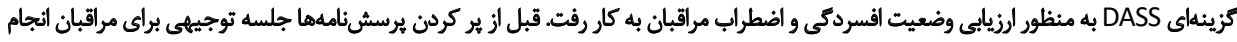

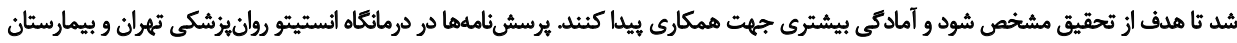

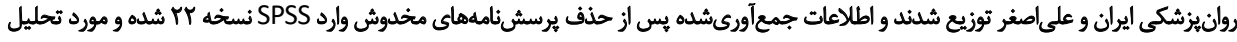

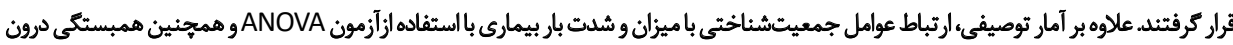

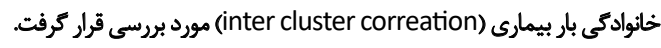

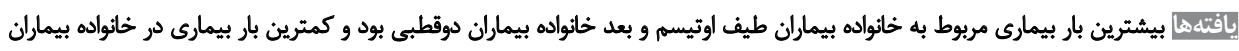

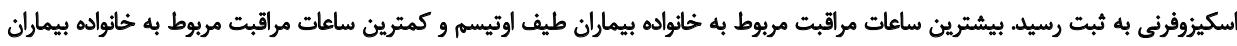

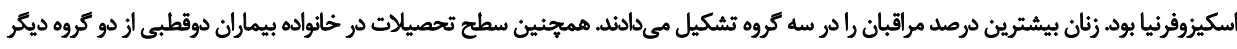

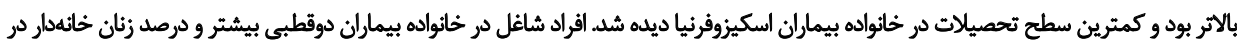

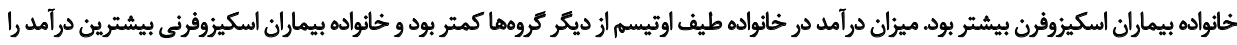

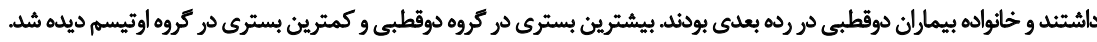

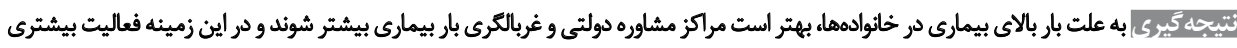

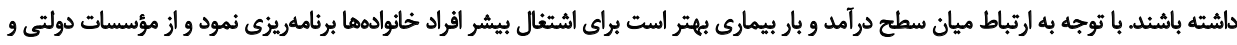

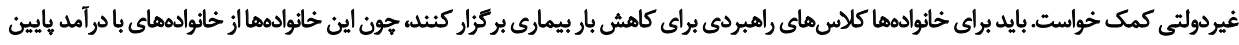

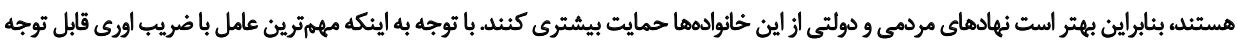

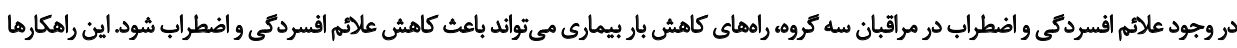

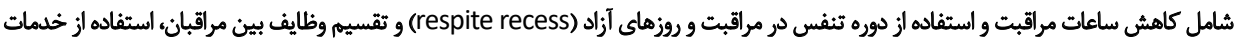

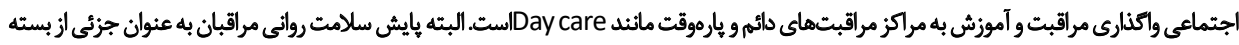

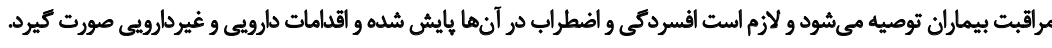

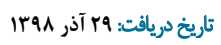

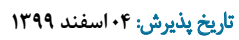

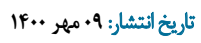

كليدوازوها:

بار مراقبت، خانواده، اسكيزوفرئيا، اختيلال

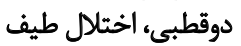
اوتيسم دوقطيc

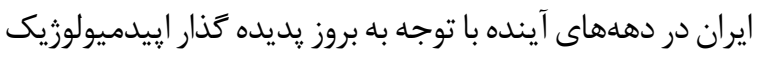

مقامه

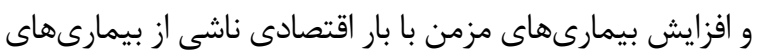

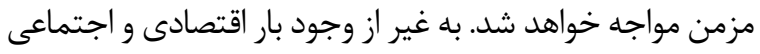
در جامعه، بيمارىهايى نظير اسكيزوفرنيا، اوتيسم و بيمارانى با بيمارىهاى غيرواگير مسئول مرگ بيش از هـ ميليون نفر در

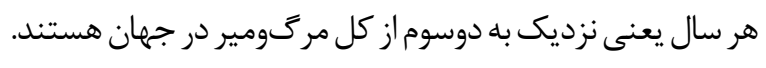

드.

$$
\begin{aligned}
& \text { "نويسنده مسئول: } \\
& \text { دكتر مسعود احمدزاده اصل }
\end{aligned}
$$

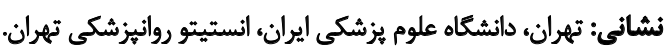

$$
\begin{aligned}
& \text { تلفن: } \\
& \text { رايانامه: ahmadshojah28@gmail.com }
\end{aligned}
$$


داروهاى ضد روانيريشى، ضد افسردگى، ليتيهم و داروهاى ضد

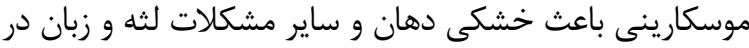
اين افراد مىشود [1.]. اختلال دوقطبى يا شيدايى افسردگى يك بيمارى مزمن همراه

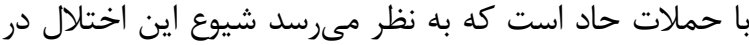

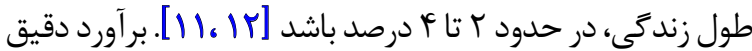

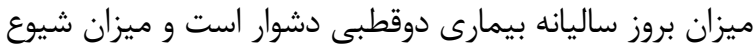

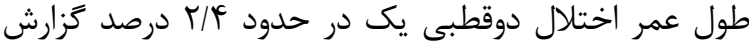

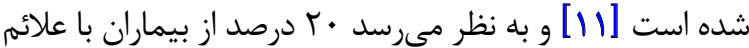

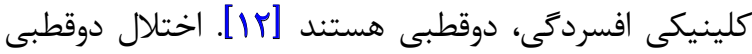

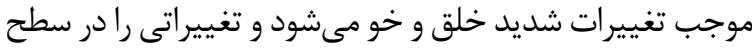

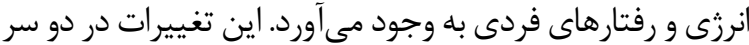

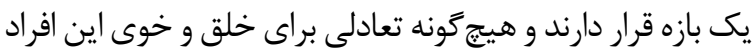

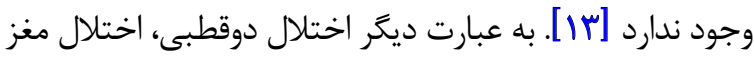

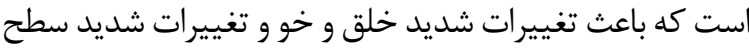

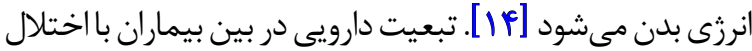

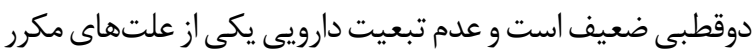

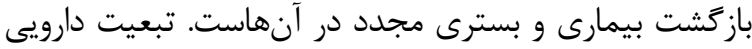

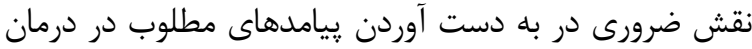

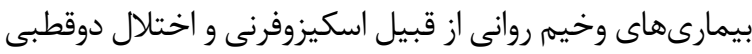

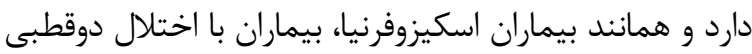

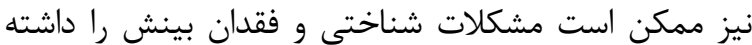

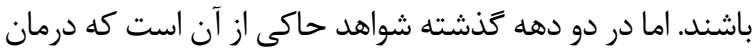

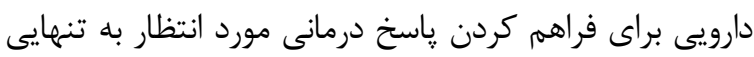

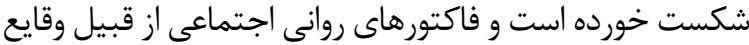

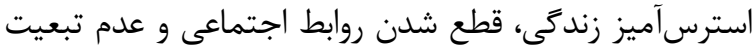

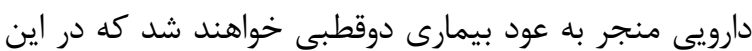

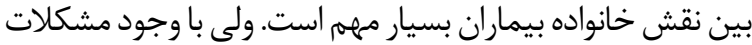

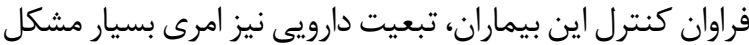

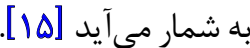
عملكرد اجرايى بيماران اسكيزوفرنيك و دوقطبى، آنكونه

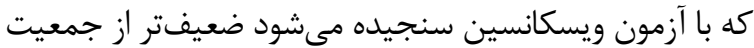

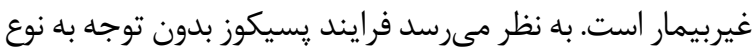

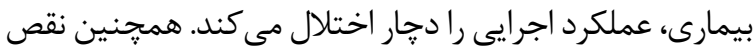

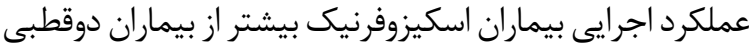

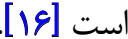

يكى از بيمارى هاى جدى از كروه بيمارىهاى رشدى اختلالات

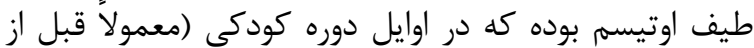

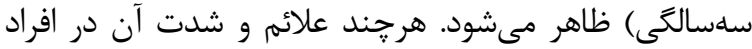

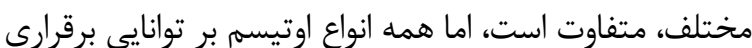

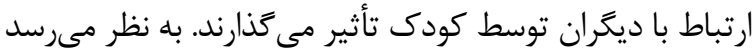

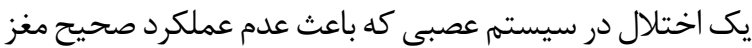

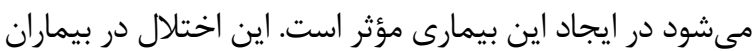

اختلال دوقطبى، بار تحملنايذيرى بر خانوادهايشان تحميل

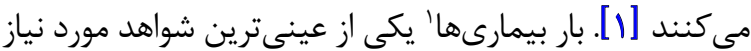

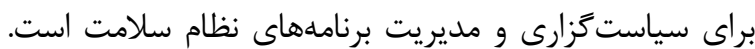

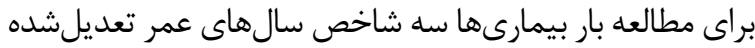

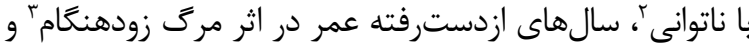

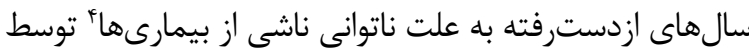

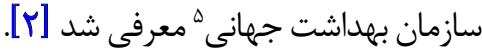
بيمارىهاى اسكيزوفرنيا، اختلال دوقطبى و اختلال اوتيسم

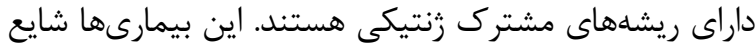

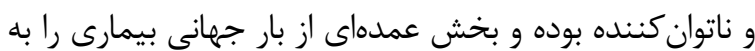

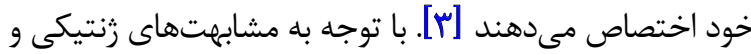

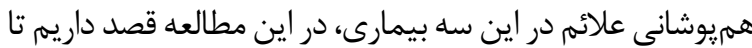

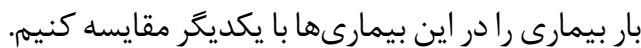
بر اساس يزوهش انجامشده زمينه فرهنكى در كشورهاى در

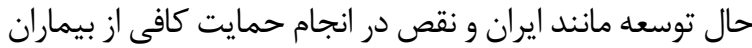

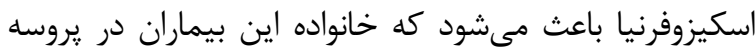

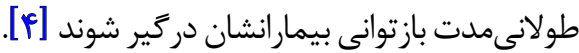
روانَسيختكى يا اسكيزوفرنى كه اكثر روانيزشكان به آن

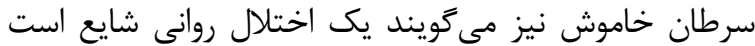

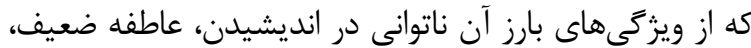

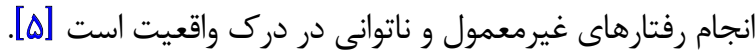

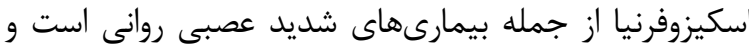

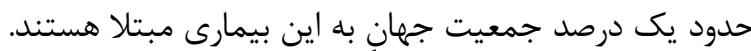

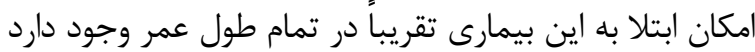

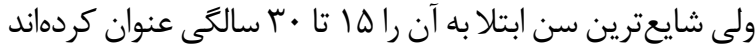

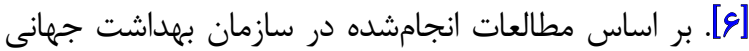

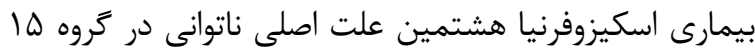

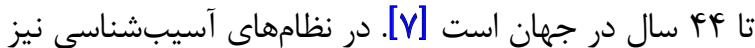

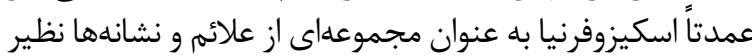

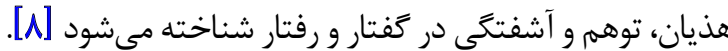
در يك يزوهش نشان داده شد بين كيفيت زندكى و راهبردهاى

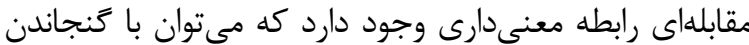

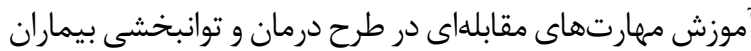

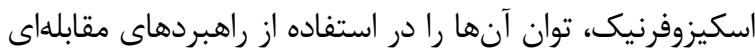

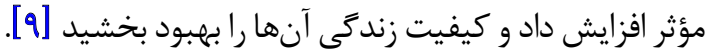
در يزوهش ديخرى نشان داده شد بيماران داراى اسكيزوفرنى

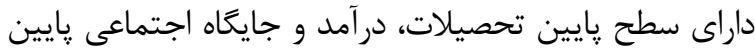

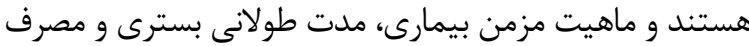

\section{Burden of diseases}

2. Disability-Adjusted Life Years (ALY)

3. Years of Life Lost (YLL)

4. Years Lived with Disability (YLD)

5. World Health Organization (WHO) 
همكارى درمانى، يذيرش بيمار از جانب خانواده، نياز به خدمات

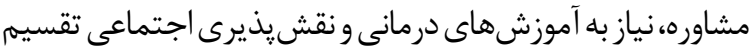

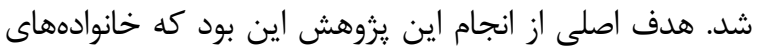

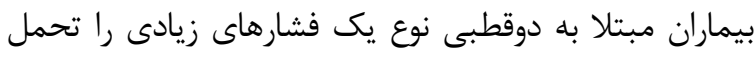

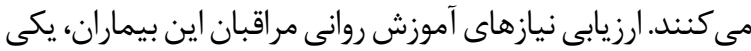

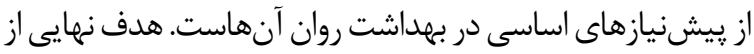

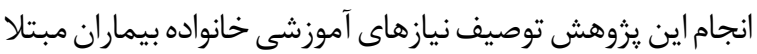

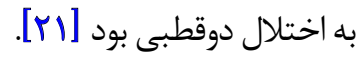
ييشينه تحقيق در خارج از ايران زانت و همكاران در يزوهش خود بيان كردند كه خانواده

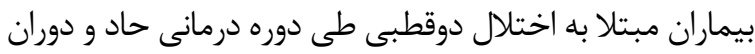

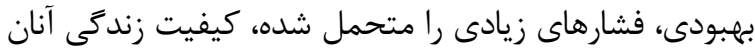

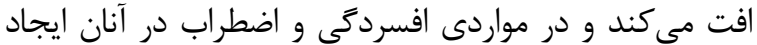

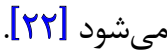

در مطالعه هادسون و همكاران نيز شواهد نشان داد مراقبين

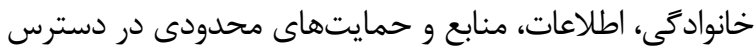

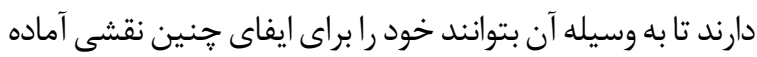

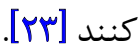

كريستجانسون و آئون در مطالعه خود به اين نتيجه رسيدند

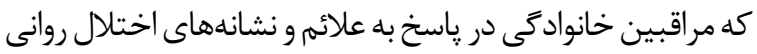

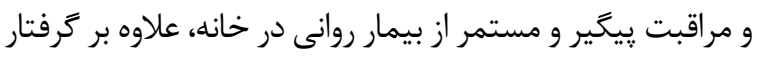

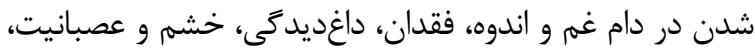

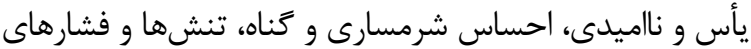

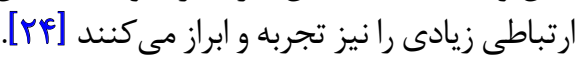

براساس يزوهش متسون و همكاران، تحقيقات موجود نشان

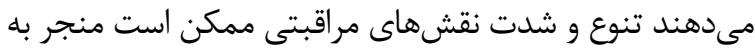

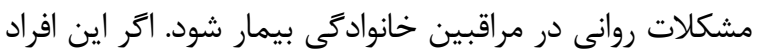

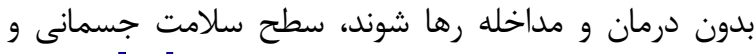

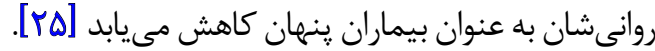
ايوى در مطالعه خود جنين نتيجه كَرفت كه بار روانى تحميلى مرئى

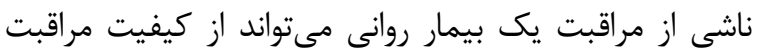

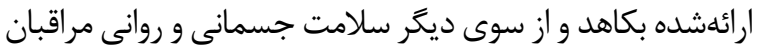

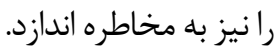

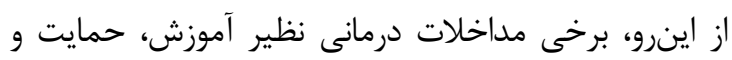

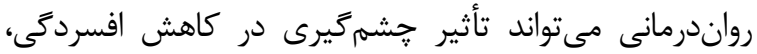

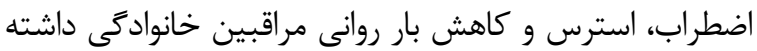

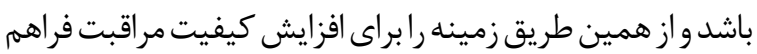

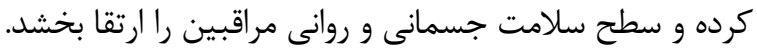

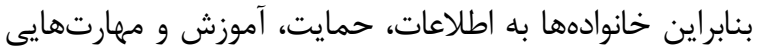

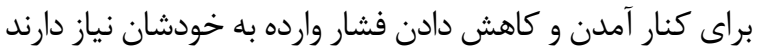

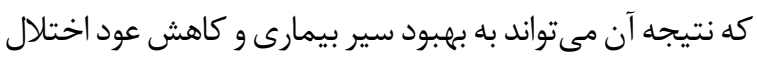

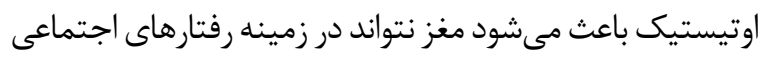

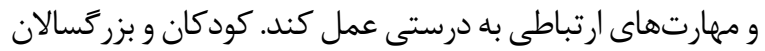

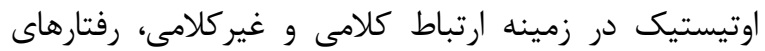

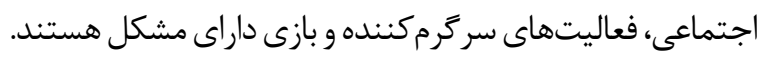

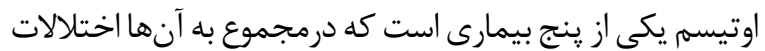
نافذ رشد (PDD) "مى كويند [IV]

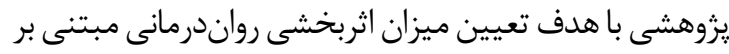

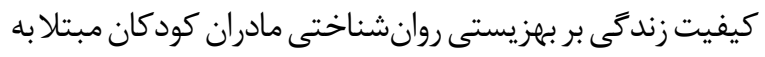

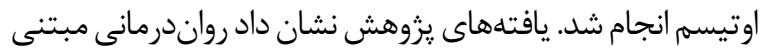

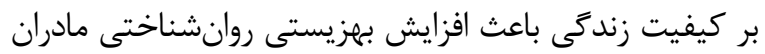

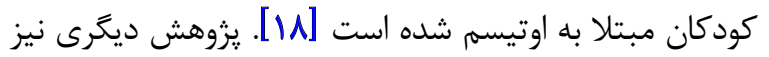

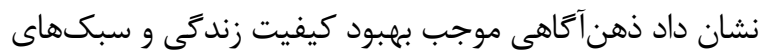

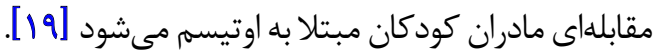

بيشينه تحقيق در ايران

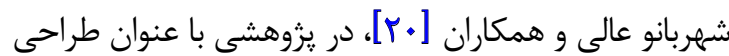

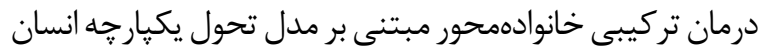

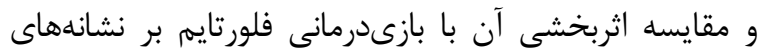

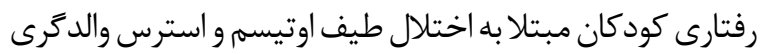

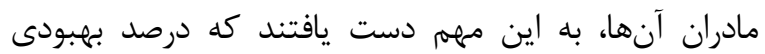

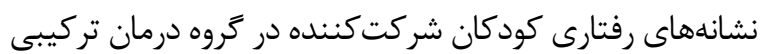

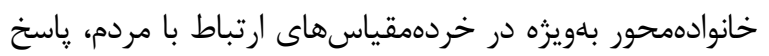

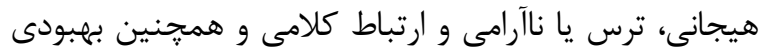

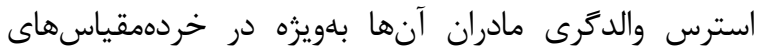

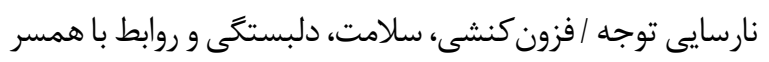

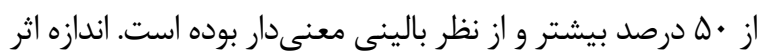

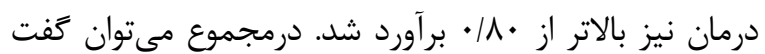

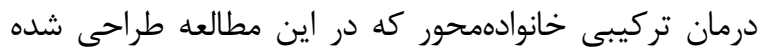

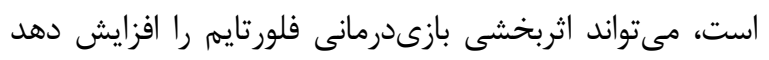

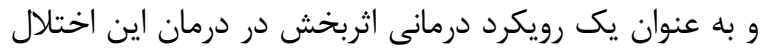

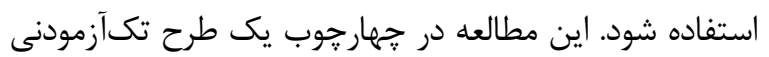

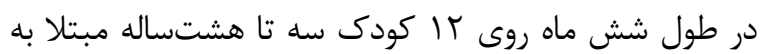

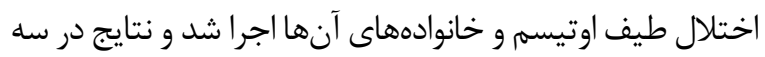

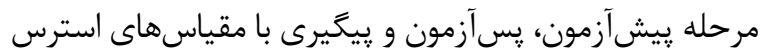

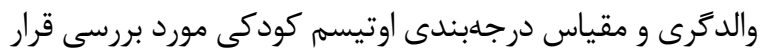

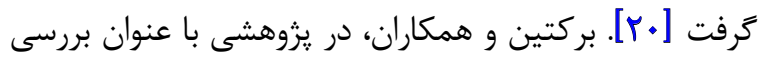

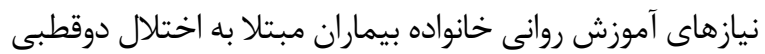

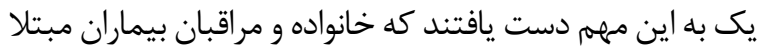

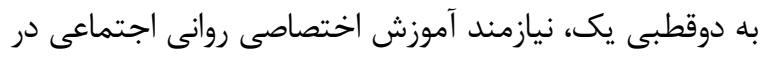

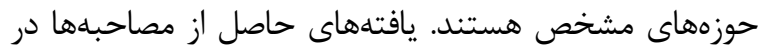

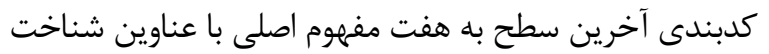
بيمارى، نحوه مقابله با مشكلات رفتارى ناشى از علائم بيمارى،

\section{Pervasive Developmental Disorders (PDD)}


مىشوند. اين بيمارىها علاوه بر فرد مبتلا افر اد مراقب بيماران را

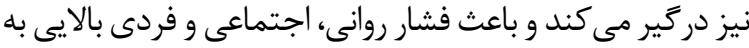

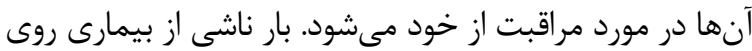

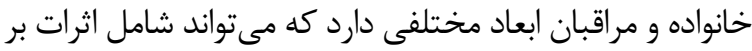

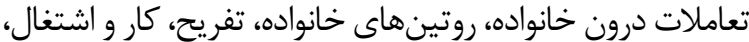

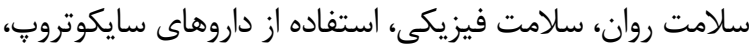

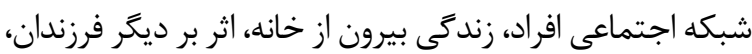

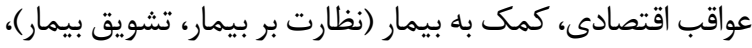

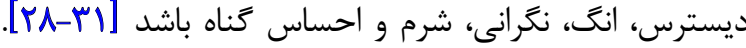

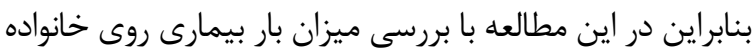

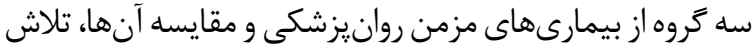

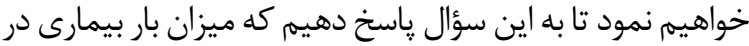

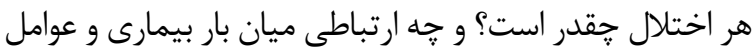

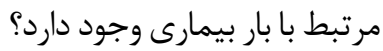

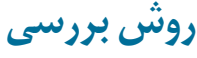

اين تحقيق از نظر نوع و هدف جزء يزوهش تهاى كاربردى و از

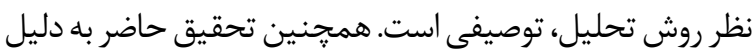

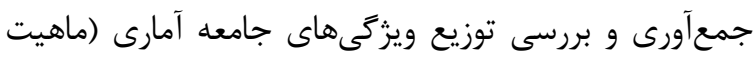

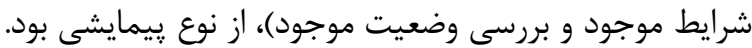

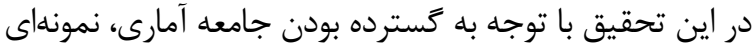

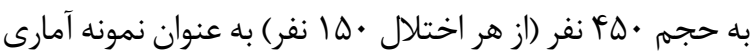

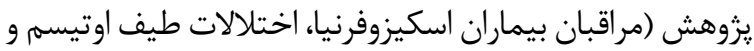

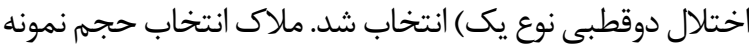

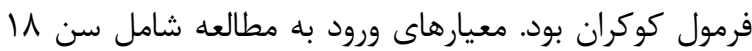

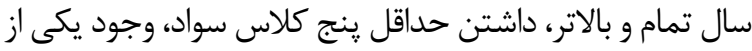

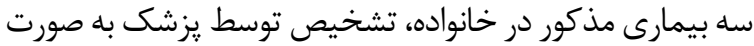

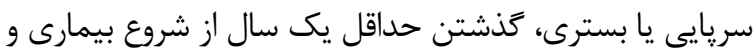

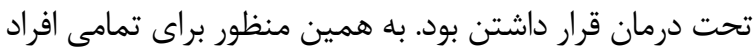

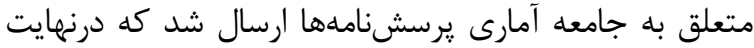

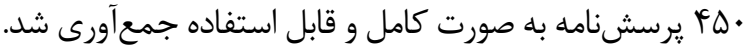

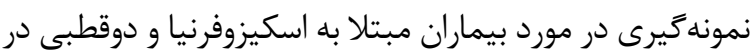

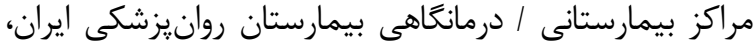

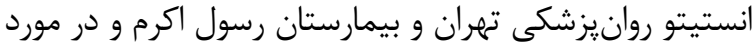

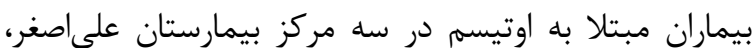

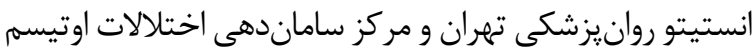

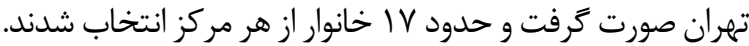

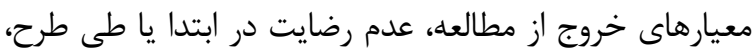

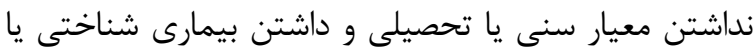

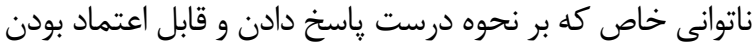

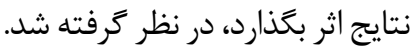

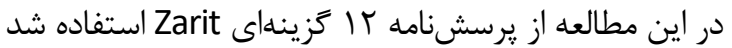

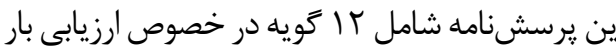

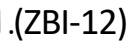

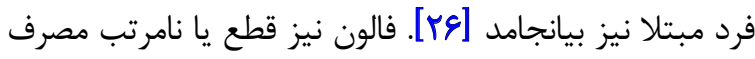

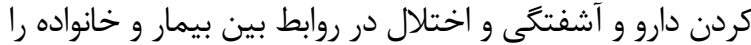

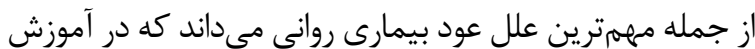

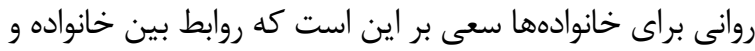

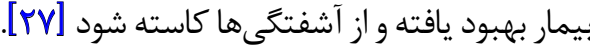

$$
\text { يرداختن به يِيشينه تحقيق }
$$

تعدادى مطالعه در مورد سنجش بار بيمارى در خانوادهها در

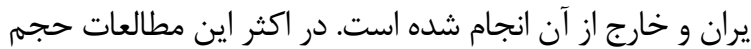

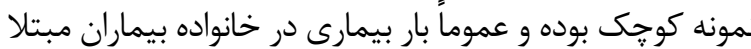

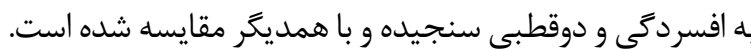

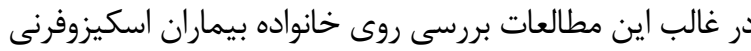

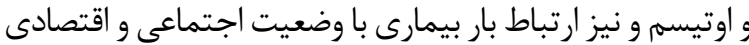

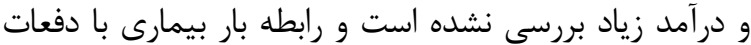

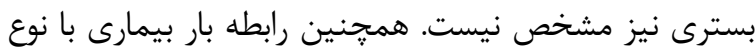

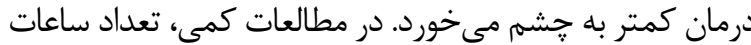

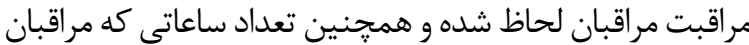

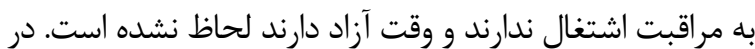

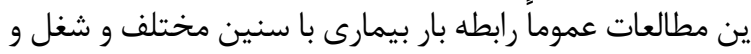
جنسيت جندان بررسى نشده است.

دلايل وجوب انجام اين مطالعه و وجه تمايز آن با مطالعات قبلى بررسى و مقايسه سه بيمارى مزمن روانيزشكى با يكديخر

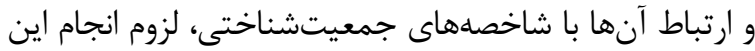

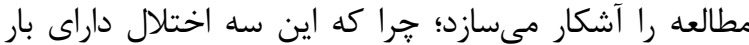

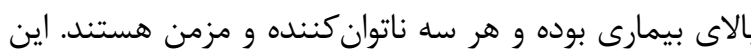

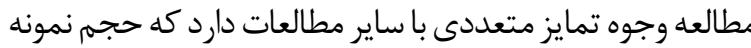

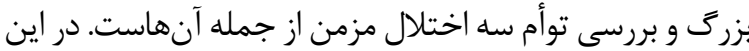

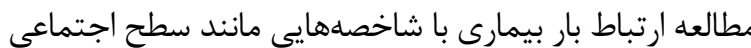
قتصادى، شغل، جنسيت، تعداد اتاقها، سطح در رآمد، تعداد نفرات آنفات

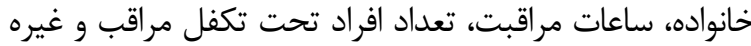

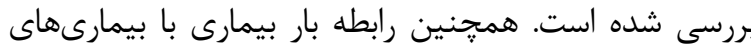

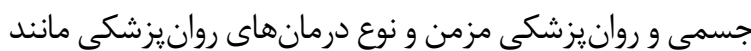

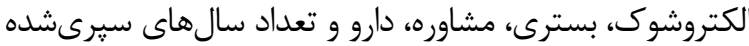

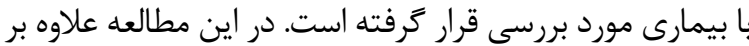

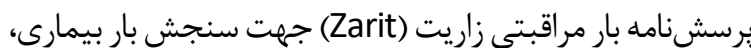

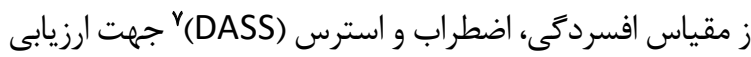
افسردگى و اضطراب بهره كرفته شده است.

بيمارىهاى شديد روانيزشكى ماندا اختلال اسكيزوفرنيا،

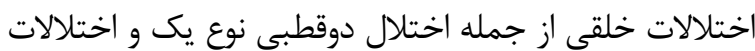

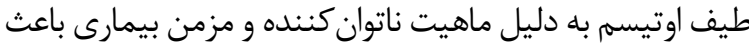

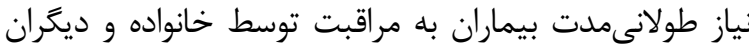

\section{Depression Anxiety Stress Scales (DASS)}




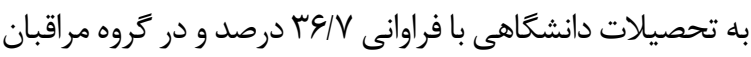

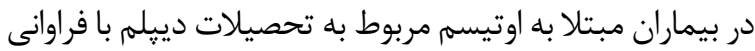

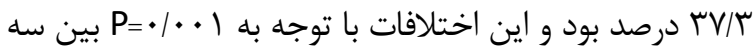

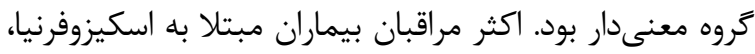

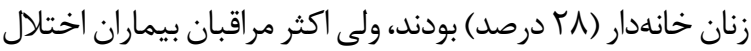

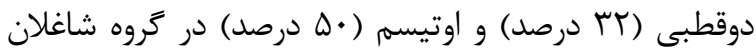

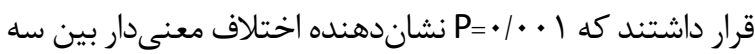

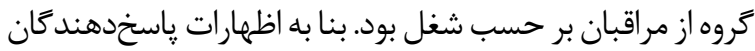

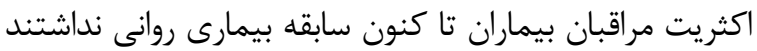

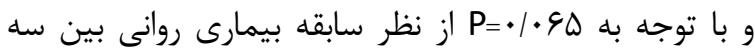

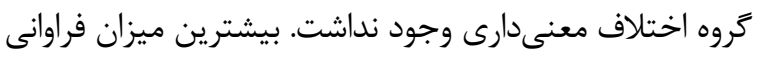

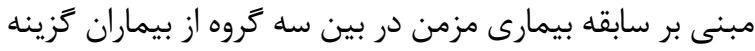

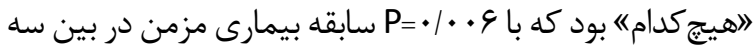

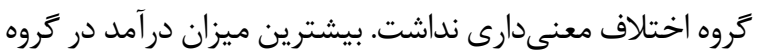

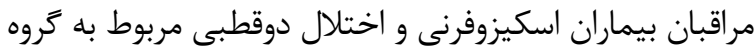

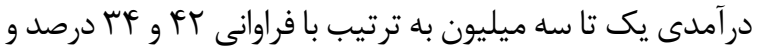

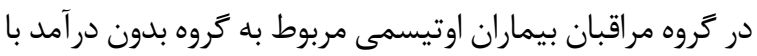

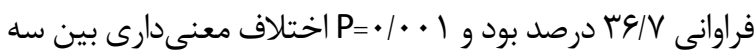

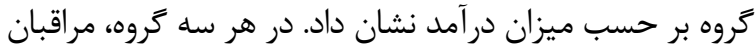

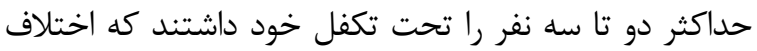

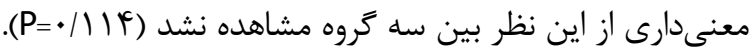

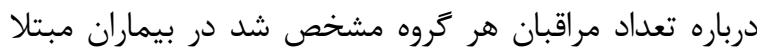

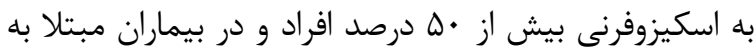

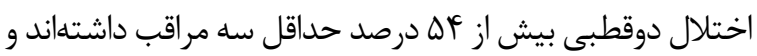

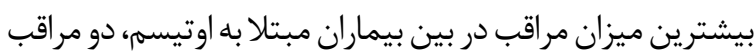

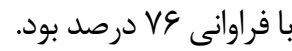

با توجه به اطلاعات بهدست آمده در جدول شماره بَ، از نظر

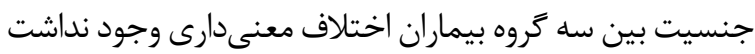

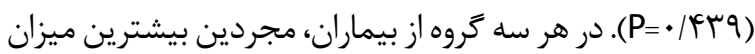

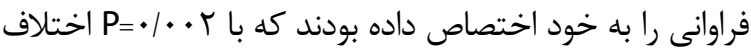

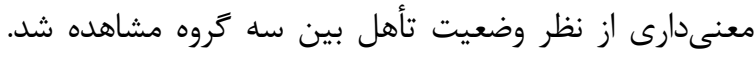

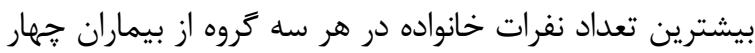

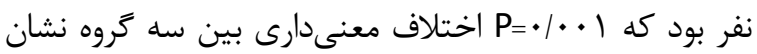

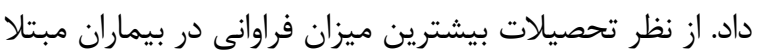

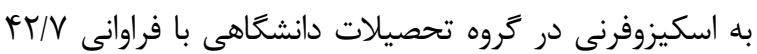

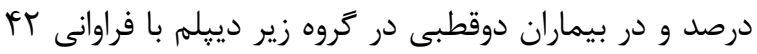

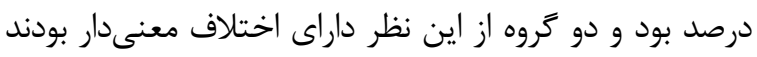

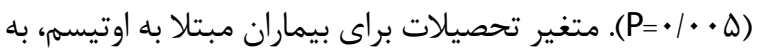

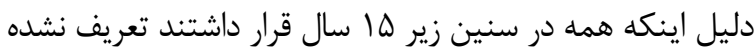

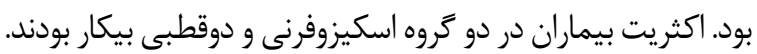

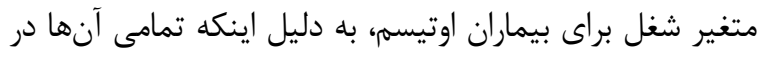

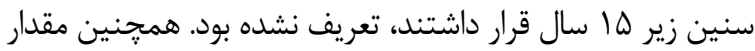

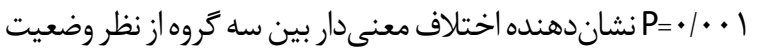

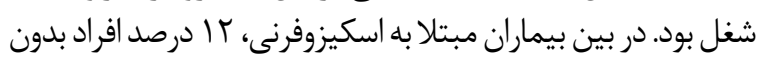

ناشى از مراقبت روى مراقبان بيماران با مقياس ليكرت صفر تا

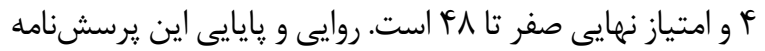

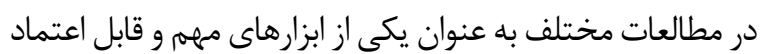

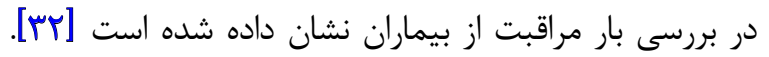

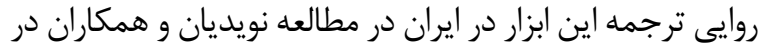

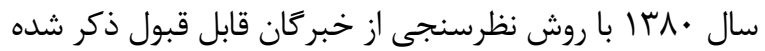

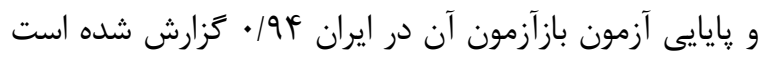

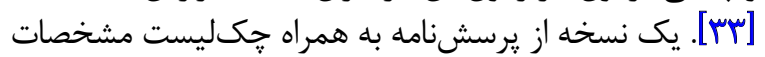

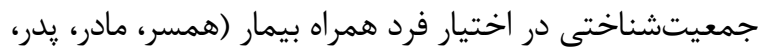

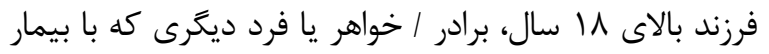

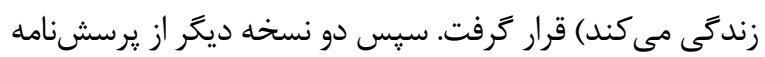

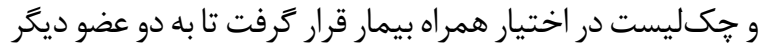

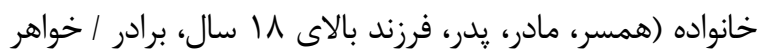

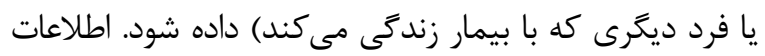

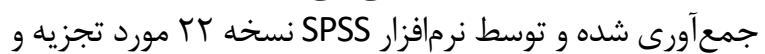
تحليل قرار كرفتند.

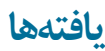

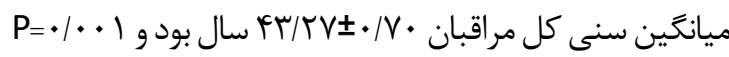

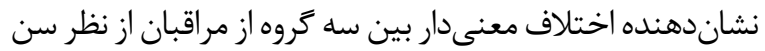

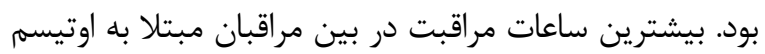

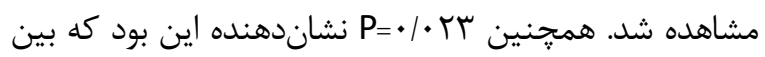

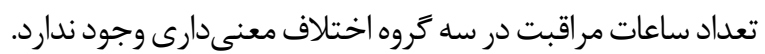

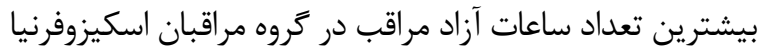

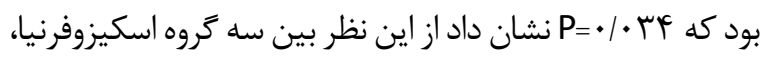

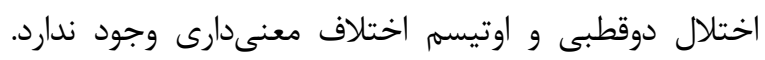

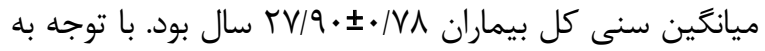

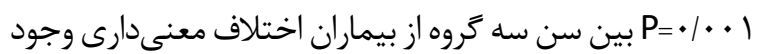

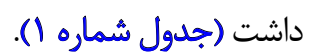

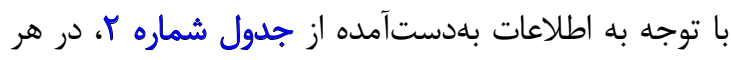

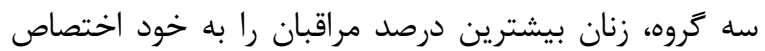

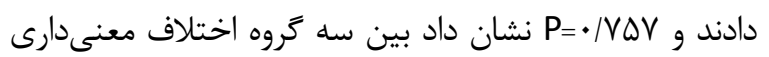

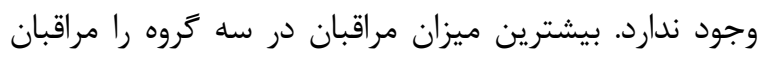

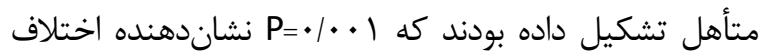

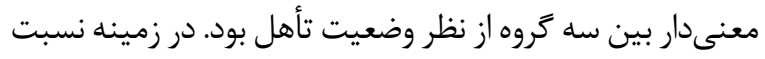

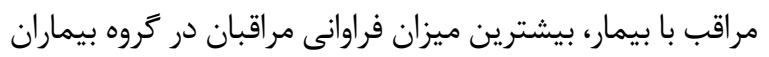

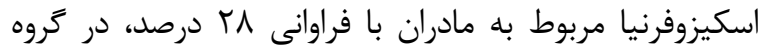

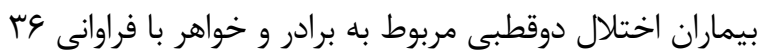

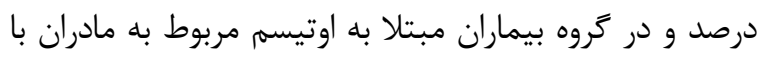

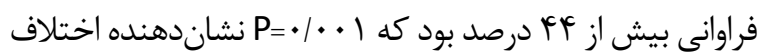

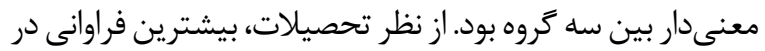

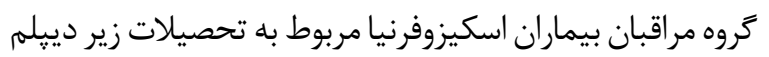

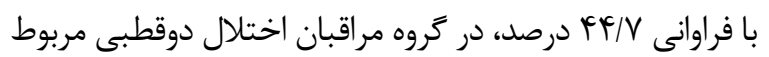


جدول ا. مقايسه ميانكين سنى و ساعات مراقبت و آزادل مراقبان و بيماران

\begin{tabular}{|c|c|c|c|c|c|}
\hline سطح معنى دارى & $\mathrm{X}^{2}$ & ميانكين +انحرافمعيار & كروهاها & \multicolumn{2}{|c|}{ متغيرها } \\
\hline & & $R T / T Y \pm V / T A$ & السكيزوفرنيا & & \\
\hline \multirow[t]{3}{*}{$<* *+\infty$} & $\pi / M^{e}$ & $P N R I \pm 1 / T Y$ & اختلال دوقطبي & سن (سال) & \\
\hline & & $r 9 / 19 \pm . / 1$ & اوتيسم & & \\
\hline & & $1 . / 8 \pi \pm \cdot / M$ & اسكيزوفرنيا & & \\
\hline \multirow[t]{3}{*}{$.1 \cdot \pi$} & V/AY & $1 . / M \pm \cdot / \& V$ & اغتلالال دوقطبيى & تعداد ساعات مراقيت & مراقبان \\
\hline & & $\mid r / / F \pm . / \Delta q$ & اوتيسم & & \\
\hline & & qr/RY $\pm \Delta / 1$. & السكيزوفرنيا & & \\
\hline \multirow[t]{3}{*}{.1 .45} & $g / M^{e}$ & $M(q) \pm \Psi / N$ & اختلالل دوقطبيى & تعداد ساعات آزاداد & \\
\hline & & $A r / \cdot r \pm r / N F$ & اوتيسم & & \\
\hline & & TV/RT $\pm V / \div 9$ & اسكيزوفرثيا & & \\
\hline \multirow[t]{2}{*}{$<+\infty \cdot \mid$} & $r Q \Delta / 1 F$ & $r \& / M \pm+/ A r$ & اختلال دوقطبى & سن (سال) & ييماران \\
\hline & & Q $/ \pm \pm \cdot / M$ & اوتيسم & & \\
\hline
\end{tabular}

Sه كه متغيرهاى مستقل توانستهاند به ترتيب بيشتر از

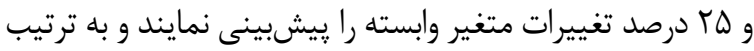
خ F خانوادههاى بيماران در كل، وضعيت روانى خانوادههاى بيماران مبتلا

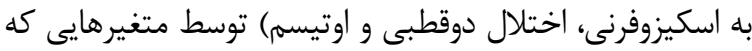

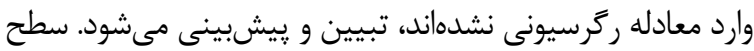

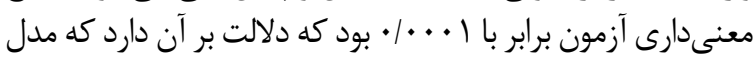

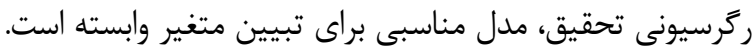

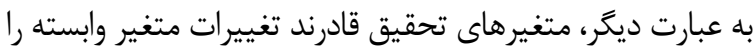

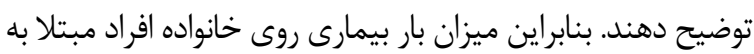

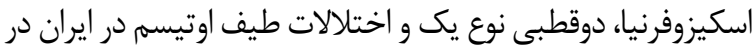

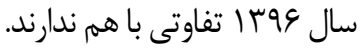

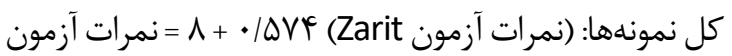
DASS اوتيسم: (نمرات آزمون Zarit) س|\&|• + N/K = نمرات آزمون DASS

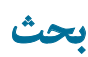

با توجه به نتايج بهدستآمده در اين مطالعه ميانگين بار بيمارى

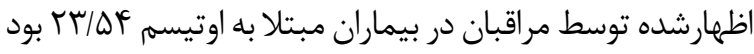

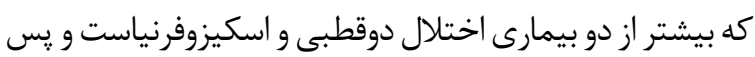

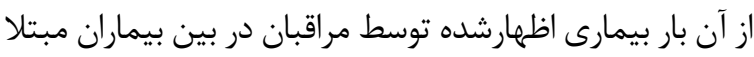

بسترى، روند درمان را ادامه داده بودند و س/11 درصد آنها بيشتر

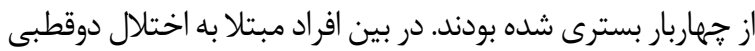

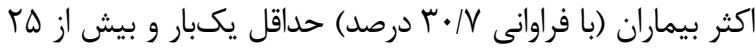

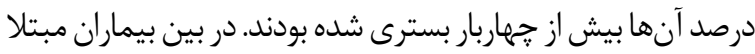

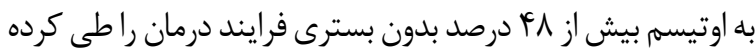

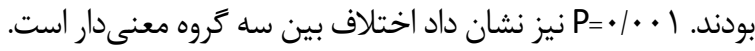
در بيماران مبتلا به اسكيزوفرنى، اختلال دوقطبى و اوتيسم بيشترين

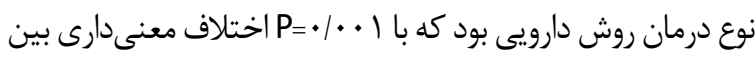

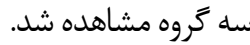

با توجه به نتايج بهدستآمده در جدول شماره f أم بيشترين ميزان

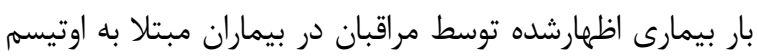

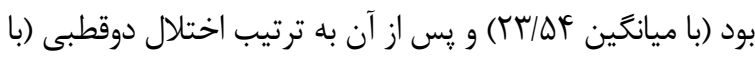

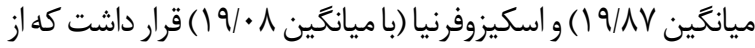

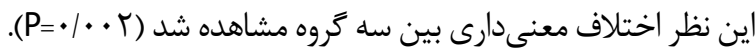

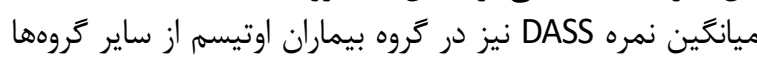

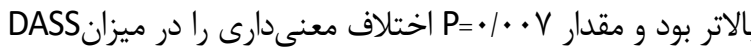

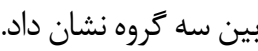
همانطور كه در جدول شماره ه ملاحظه مىشود، ضريب همبستكى خطى براى تبيين وضعيت روانى خانوادههاى بيماران

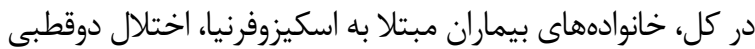

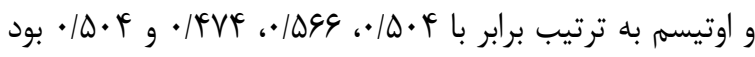

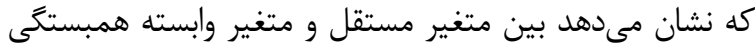

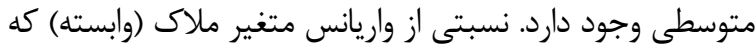

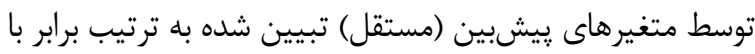


جدول ب. مقايسه ويزُكى هاي جمعيتشئاختى مراقبان

\begin{tabular}{|c|c|c|c|c|c|c|}
\hline \multirow{2}{*}{ سطح معنى دارى } & \multirow{2}{*}{$x^{2}$} & \multicolumn{3}{|c|}{ درصد } & \multirow{2}{*}{\multicolumn{2}{|c|}{ متغيرها }} \\
\hline & & اوتيسم & اختلال دوقطبي & اسكيزوفرنيا & & \\
\hline \multirow{3}{*}{ - Nar } & \multirow{3}{*}{.$/ \Delta \Delta A$} & $F \Delta / \Psi$ & $\pi$ & 48 & مرد مر & \multirow{3}{*}{ جنسيت } \\
\hline & & & & & & \\
\hline & & $\Delta \varphi / N$ & $\Delta A$ & $\Delta)^{p}$ & is & \\
\hline \multirow{5}{*}{$<+1++1$} & \multirow{5}{*}{$P \Delta / \Delta Y$} & 9. & $\Delta$ & $n / / r$ & مثأهل & \multirow{5}{*}{ وضعيث تأهل } \\
\hline & & e/V & $W / \mu$ & $\pi / \pi$ & مجرد & \\
\hline & & & & & & \\
\hline & & $r$ & $r / T$ & $m / r$ & بيوه & \\
\hline & & $1 / r$ & $r / T$ & r & مطلقه & \\
\hline \multirow{5}{*}{$<+1++1$} & \multirow{5}{*}{ IFNG. } & 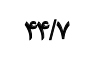 & $r+/ Y$ & rA & 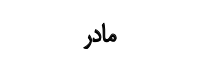 & \multirow{5}{*}{ نسبث } \\
\hline & & f. & 18 & re & يلر & \\
\hline & & $\wedge$ & ( & rA & برادر و خواهر & \\
\hline & & * & if & $q / 4$ & فرزئد & \\
\hline & & $V / r^{\mu}$ & $\mid w / m$ & $1 . N$ & ساير س & \\
\hline \multirow{5}{*}{.$\% 1$} & \multirow{5}{*}{$W / \pi r$} & re & $\pi / N$ & $\operatorname{pep}$ & زير دييلم & \multirow{5}{*}{ تحصيلات } \\
\hline & & $r / T$ & TNY & $\pi$ & دييلم & \\
\hline & & r. & TEN & $r \cdot N$ & مانشكاهى & \\
\hline & & $\mathscr{E N}$ & $1 / r$ & $1 / r$ & دكترى & \\
\hline & & - &.$/ Y$ & $1 / 4$ & هيجكدام & \\
\hline \multirow{6}{*}{$<\cdot 1 . .1$} & \multirow{6}{*}{ EV/a. } & 8 & $\mid w / r$ & $V / r$ & بيكار & \multirow{6}{*}{ شُغل } \\
\hline & & Q. & $\pi$ & $r / T$ & 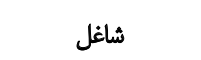 & \\
\hline & & $r / Y$ & $\mid w / m$ & $r e / v$ & باز نشسته & \\
\hline & & $r / Y$ & 1. & $q / \mu$ & دانشحي & \\
\hline & & $\Gamma \Delta / \mu$ & rA & rA & خائه دار & \\
\hline & & L & $r / \varphi$ & $r / \varphi$ & ساير & \\
\hline \multirow{3}{*}{.1 .90} & \multirow{3}{*}{ NAF } & $\wedge$ & $1 . / N$ & PN & بله & \multirow{3}{*}{ سابقه بييمارى رواثى } \\
\hline & & ar & wr & $9 \% / N$ & خير & \\
\hline & & - &.$/ Y$ & $r / V$ & مايل به اظهلار نيستم. & \\
\hline \multirow{5}{*}{.10 .8} & \multirow{5}{*}{$M / M$} & if & r & 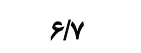 & بيمارى جسمى & \multirow{5}{*}{ سابقه ييمارى مزمن } \\
\hline & & if & $r+/ V$ & $r r / V$ & بيمارى روائى & \\
\hline & & 8 & $1 . / V$ & $W / r$ & هر دو & \\
\hline & & $\Delta N Y$ & $H / T$ & $\Delta V / /$ & هيجكدام & \\
\hline & & $v / r$ & $\Delta / r$ & $\wedge$ & مايل به اظهلار نيستم. & \\
\hline
\end{tabular}




\begin{tabular}{|c|c|c|c|c|c|c|}
\hline \multirow{2}{*}{ سطح معنى دارى } & \multirow{2}{*}{$\mathrm{X}^{2}$} & \multicolumn{3}{|c|}{ درصد } & \multirow{2}{*}{\multicolumn{2}{|c|}{ مثغيرها }} \\
\hline & & اوتيسم & اختّلال دوقطبى & اسكيزوفرنيا & & \\
\hline \multirow{6}{*}{$<+1+\infty$} & \multirow{6}{*}{ FV/AS } & 1. & $r+N$ & ir & زير يك ميليون & \multirow{6}{*}{ لدرآمد } \\
\hline & & rA & $m$ & rr & يك تا سه ميليون & \\
\hline & & if & $\wedge$ & r & سه تا ينج ميليون & \\
\hline & & 8 & $1 / \pi$ & $r / \mu$ & بالاى ينج ميليون & \\
\hline & & TEN & $r \cdot / r$ & $r T / N$ & بدون در أمد & \\
\hline & & $\Delta / r$ & $10 / \Psi$ & $\wedge$ & مايل به باسخ كويى نيستم. & \\
\hline \multirow{6}{*}{.$M F^{\circ}$} & \multirow{6}{*}{$r \Delta / \pi T$} & $1 / 0$ & g/A & V/e & ليك نثر & \multirow{6}{*}{ افراد تحت تكفل } \\
\hline & & $r / r$ & ro & $m e / p$ & دو نفر & \\
\hline & & $\Delta . / N$ & $r \Delta / \Delta$ & $m e / f$ & بسه نفر & \\
\hline & & $1 \% / F$ & INT & M & جهار نفر & \\
\hline & & $1 / \Delta$ & $r / \Delta$ & V/A & بيشتر & \\
\hline & & $1 / 0$ & - & V/F & مايل به ياسختكويى & \\
\hline \multirow{5}{*}{$<+\mid+1$} & \multirow{5}{*}{$W / 18$} & ve & $\pi / N$ & pif & دو نفر & \multirow{5}{*}{ تعلداد مراقبان بيمار } \\
\hline & & 18 & $\Delta F / N$ & $\Delta \cdot N$ & لمه نفر & \\
\hline & & & & & & \\
\hline & & $F / N$ & NV & $\Delta / \pi$ & جهارثفر & \\
\hline & & $r / r$ & $r$ & - & بيشتر & \\
\hline
\end{tabular}

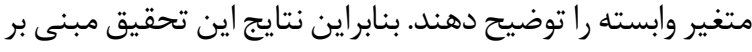

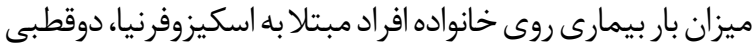

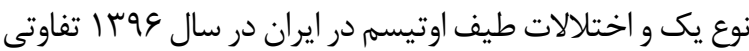

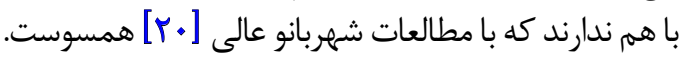

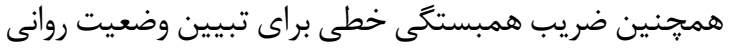

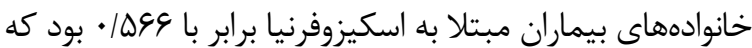
نشان مىدهد بين متغير مستقل و متغير وابسته همبستيتى إنى

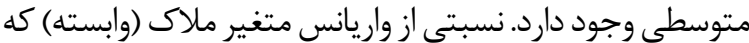

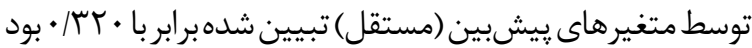

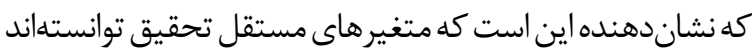

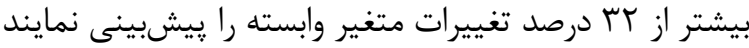

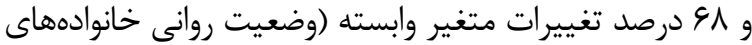

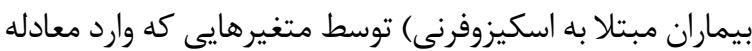

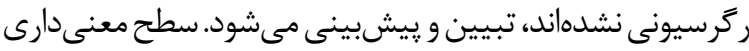

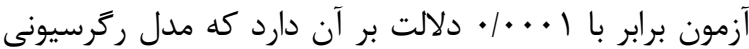

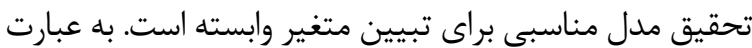

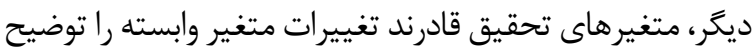
دهند. اين نتايج با تحقيقات نويديان و همكاران [بآس] همسوست.
به اختلال دوقطبى بيشتر از بيماران مبتلا به اسكيزوفرنيا بود.

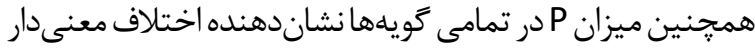

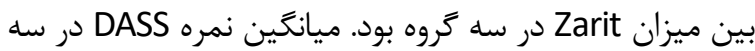

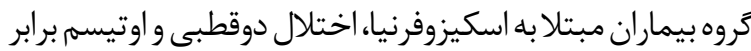
با را از اين نظر بين سه كروه نشان داد.

نتايج اين مطالعه نشان دادضر يب همبستكى خطى براى تبيين

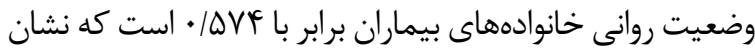

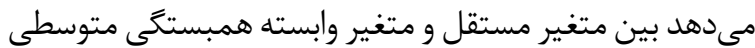

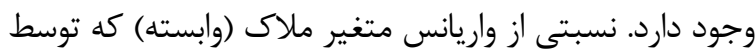

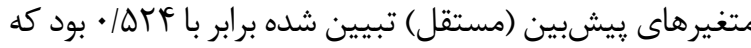

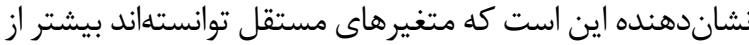

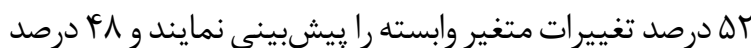

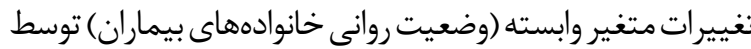

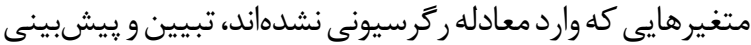

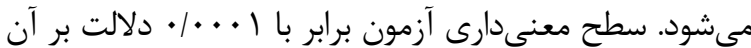

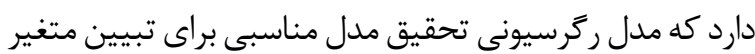
وابسته استبه عبارت ديكر، متغيرهاى تحقيق قدانيق تادرند تغييرات 
جدول لا. مثايسه ويرُكى هاي جمعيتشئاختى بيماران

\begin{tabular}{|c|c|c|c|c|c|c|}
\hline \multirow{2}{*}{ سطح معنى دارى } & \multirow{2}{*}{$x^{2}$} & \multicolumn{3}{|c|}{ مرصد } & \multirow{2}{*}{ متغيرها } & \\
\hline & & اوتيسم & اختلال دوقطبى & اسكيزوفرنيا & & \\
\hline \multirow{3}{*}{. /Frq } & \multirow{3}{*}{ V/gt } & f. & 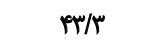 & $\mathrm{F} / \mathrm{T}$ & هرد & \multirow{3}{*}{ جئسيت } \\
\hline & & & & & & \\
\hline & & q. & $\Delta S N$ & $\Delta T / N$ & زن & \\
\hline \multirow{5}{*}{ /..r } & \multirow{5}{*}{$1 Q / I T$} & . & ג & r. & متأهل & \multirow{5}{*}{ وضعيت تأهل } \\
\hline & & $1 .$. & QPR & $r / N$ & مجرد & \\
\hline & & & & & & \\
\hline & & . & $\Delta / r$ & $T / N$ & بيوه & \\
\hline & & - & r & $F / Y$ & مطلقه & \\
\hline \multirow{8}{*}{$<* 1 .+1$} & \multirow{8}{*}{$\Delta \Delta / V \Delta$} & . & - & $T / Y$ & Y نفر & \multirow{8}{*}{ ثلعلاد نفراده } \\
\hline & & rA & r. & Mf & بتنر & \\
\hline & & $P V / T$ & $p / x$ & TNY & بن نفر & \\
\hline & & $\mid V / r$ & M & M & ه نفر & \\
\hline & & 8 & $\mid v / \pi$ & $1 . N$ & ونفر & \\
\hline & & $1 / r$ & r/ & $1 . / N$ & V نقر & \\
\hline & & . & . & $1 / r$ & هنفر & \\
\hline & & . & . & p & ا انفر & \\
\hline \multirow{5}{*}{100} & \multirow{5}{*}{$18 / 99$} & - & $r / r$ & $\wedge$ & ميىسواد & \multirow{5}{*}{ تحصيلات } \\
\hline & & - & rit & $r q / r$ & زير دييلم & \\
\hline & & & & & & \\
\hline & & - & $\mathrm{rV} / \mathrm{T}$ & $r T / Y$ & كانشكاهى & \\
\hline & & - & $\mathrm{rv} / \mathrm{r}$ & r. & دكترى & \\
\hline \multirow{5}{*}{$<+/+\infty$} & \multirow{5}{*}{$r r / q \gamma$} & - & $\Delta V / T$ & ENY & بيكار & \multirow{5}{*}{ شغل } \\
\hline & & - & $q / r$ & $\wedge$ & شاغل & \\
\hline & & - & $11 / \%$ & . & باز نشسته & \\
\hline & & - & $r$ & $\Delta / r$ & كائشجو & \\
\hline & & - & $\mid V / \pi$ & M & خانهدار & \\
\hline \multirow{6}{*}{$<* / . .1$} & \multirow{6}{*}{ IFITA } & PNY & $p$ & ir & بدون بسترى & \multirow{6}{*}{ تعبداد دفعات } \\
\hline & & TEN & $r \cdot N$ & $r \cdot / V$ & يك بار & \\
\hline & & 1. & $I E N$ & in & دوبار & \\
\hline & & f & $W / \pi$ & if & ل مبه بار & \\
\hline & & SN & ir & if & جهار بار & \\
\hline & & $i$ & $r \Delta / T$ & $11 / \pi$ & ييشتر & \\
\hline
\end{tabular}




\begin{tabular}{|c|c|c|c|c|c|c|}
\hline \multirow{2}{*}{ سطح معنى دارى } & \multirow{2}{*}{$x^{2}$} & \multicolumn{3}{|c|}{ درصد } & \multirow{2}{*}{ متغيرها } & \\
\hline & & اوتيسم & اختلال دوقطبي & اسكيزوفرنيا & & \\
\hline \multirow{9}{*}{$<+1++1$} & \multirow{9}{*}{$W E / M$} & $\mathrm{r} / / \mathrm{T}$ & $n / m$ & $1 \mathrm{M} / \mathrm{r}$ & دارويى & \\
\hline & & $\Delta / r$ & 8 & - & مشاوره & \\
\hline & & $1 . / N$ & - & • & كاردرمانى & \\
\hline & & $1 / \pi$ & - & - & ساير & \\
\hline & & • & • & $T / V$ & شوكى و دارو & نوع درمان \\
\hline & & $r \cdot / V$ & r & + & دلارو، مشاوره وكاردرمانى & \\
\hline & & 19 & $10 / \%$ & $\wedge$ & مارو و هشاوره & \\
\hline & & $r \cdot N$ & $r / r$ & - & دارو و كاردرمائى & \\
\hline & & - & - & $g / N$ & شوك، دارو، كاردرمانى و مشاوره & \\
\hline
\end{tabular}

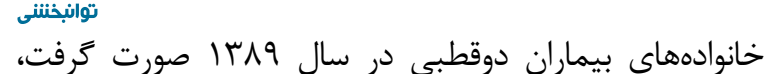

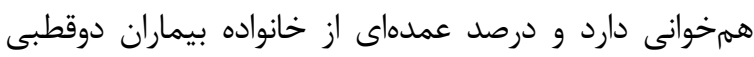

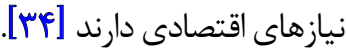

همجنين در يزوهشى كه در بيماران اسكيزوفرنى بسترى

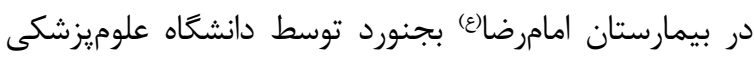

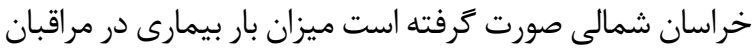

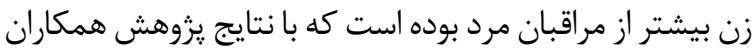

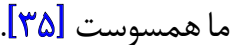

نتايج مطالعهاى كه در مورد بررسى مقايسهاى بار بيمارى

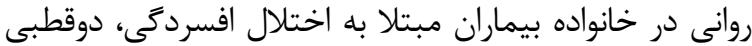

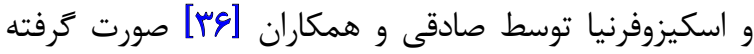

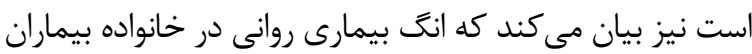

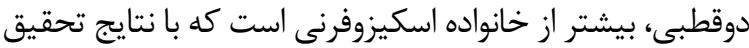

ما ماهمسوست.

همجنين نتايج نشان داد ضر يب همبستخى خطى براى تبيين

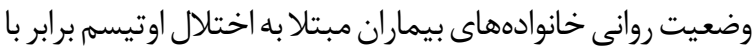

نتايج تحقيق كنونى حاكى از آن است كه ضريب همبستكى

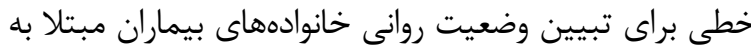

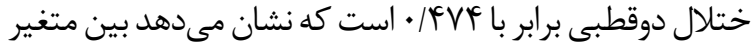

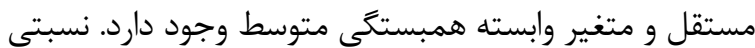

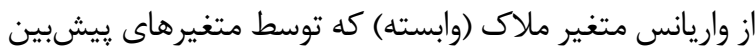

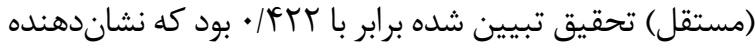

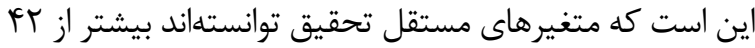

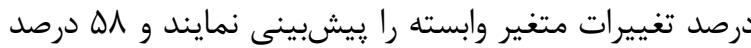

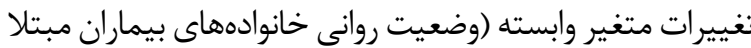
به اختلال دوقطبى) توسط متغيرهايى كه وارد معادله ركر سيونى نئي

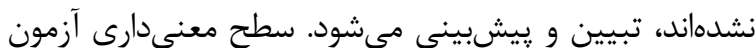

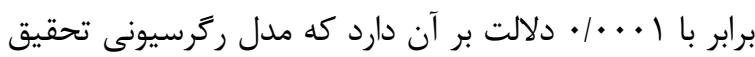

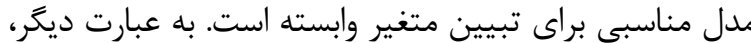

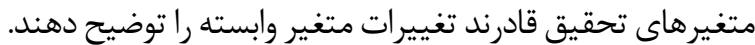

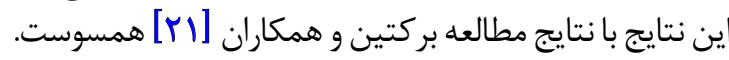

نتايج حاصل از اين مطالعه در زمينه نيازهاى اقتصادى، با

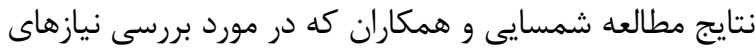

جدول ع. مقايسه ميزان بار بيمارى و ميزان افسردكى و اضطراب مراقبين در سه كروه

\begin{tabular}{|c|c|c|c|c|}
\hline سطح معنى دارى & $x^{2}$ & مياتكيندانحرافمعيار & كروهها & 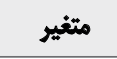 \\
\hline \multirow{3}{*}{$+1 /+r$} & \multirow{3}{*}{$1 T / 4 F$} & $1 \vee \cdot \Lambda \pm 1 r / A$ & اسكيزوفرنيا & \multirow{3}{*}{ Zarit } \\
\hline & & $19 / N A \pm I T / M 9$ & أختالال دوقطبيى & \\
\hline & & $r T / \Delta F \pm I V / r$ & الوتيسم & \\
\hline \multirow{3}{*}{ 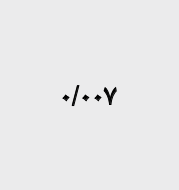 } & \multirow{3}{*}{ V/AT } & WretIF/Tr & اسكيزوفرنيا & \multirow{3}{*}{ DASS Dمره D } \\
\hline & & Wra $\pm I r / A V$ & اختالال دوقطبى & \\
\hline & & $T r / q) \pm \mid \Delta / .$. & الوتيسم & \\
\hline
\end{tabular}


جدول ه. نتايج ركرسيون خطى براي كل نمونهها و بيماران مبتلا به اسكيزوفرئيا، اختلال دوقطبى و اوتيسم

\begin{tabular}{|c|c|c|c|c|c|c|}
\hline سطح معنى دارى & $\mathbf{t}$ & Beta & SE & B & مدل & بيمارى \\
\hline$<\cdot 1 \cdot .1$ & $V / \cdot 18$ & & VIT & V/ares & مقدار ثابت & \\
\hline$<+1+.1$ & IT/Feg & $+/ \Delta+F$ & $+1+48$ & $\cdot / \Delta V^{e}$ & Zarit نمرات آزمون & كل نمونهها \\
\hline
\end{tabular}

$\operatorname{AdR}^{2}=\cdot / \Delta \cdot+$

$R^{2}=/ \Delta T r$

$R=+/ \Delta+f$

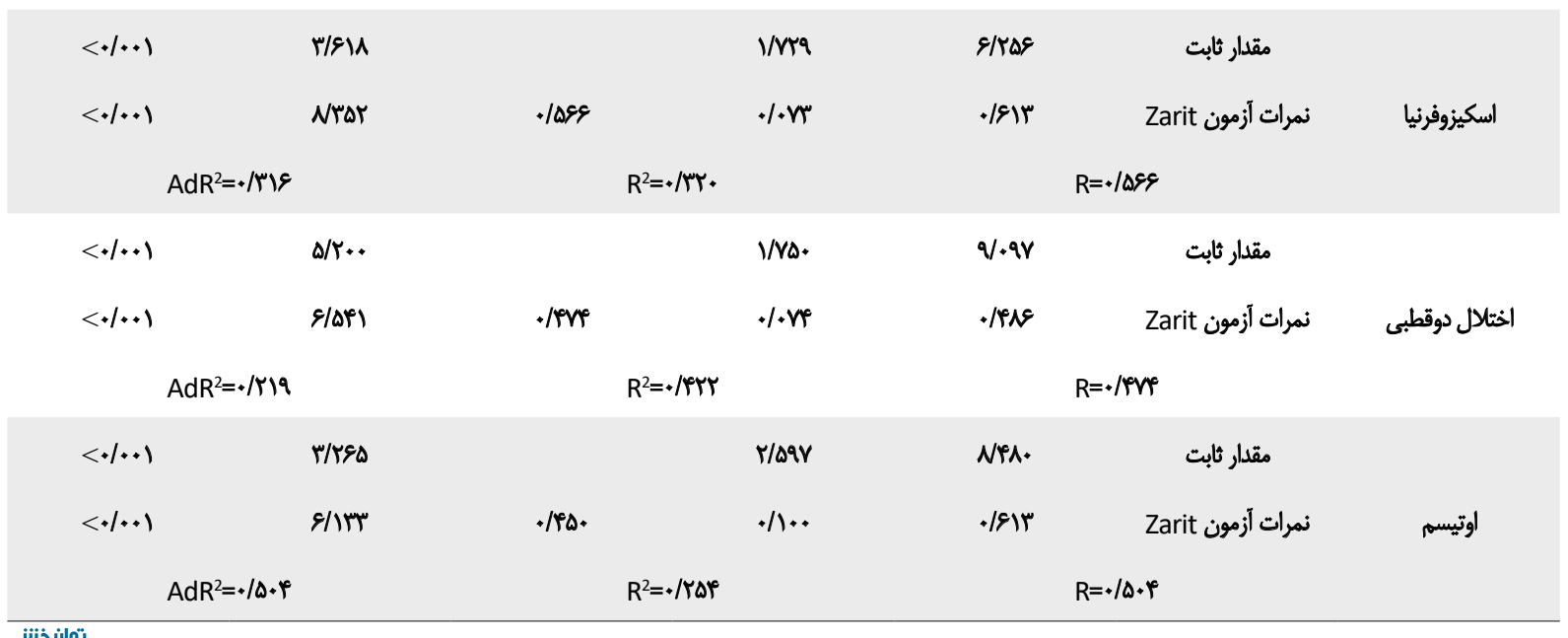

راهبردى براى كاهش بار بيمارى بر گزار كنند، جون در تحقيق

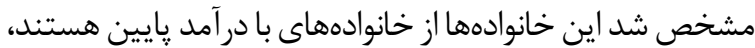

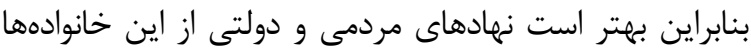

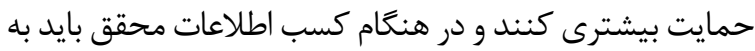

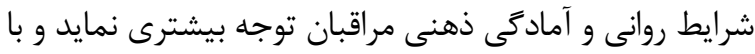

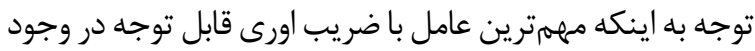

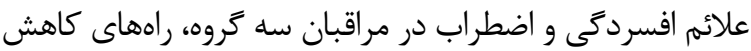

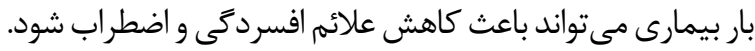

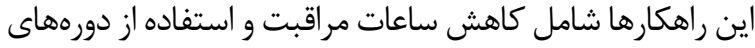

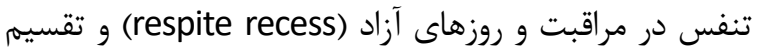

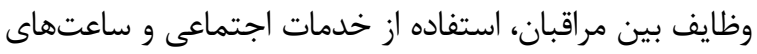

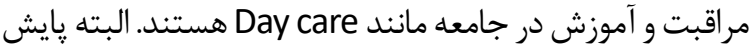

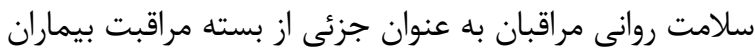

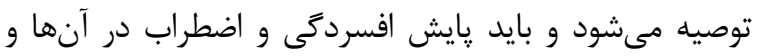

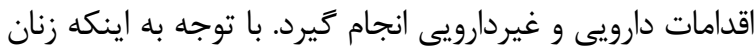

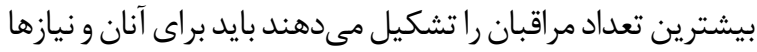

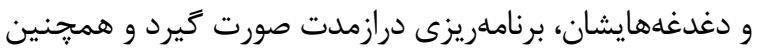

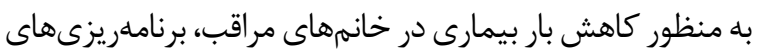

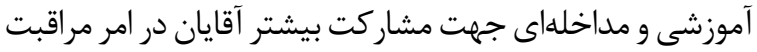

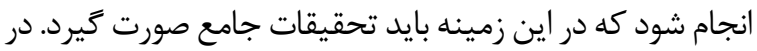

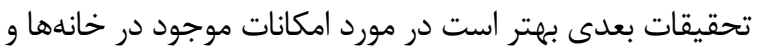

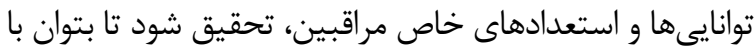

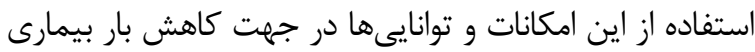

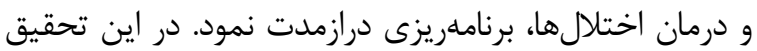

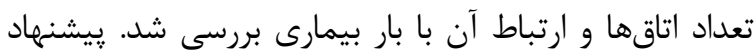

F • • • • است كه نشان مى دهد بين متغير مستقل و متغير وابسته

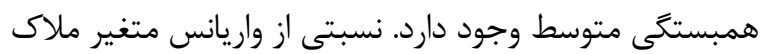

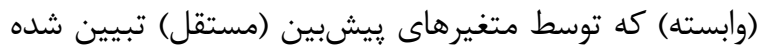

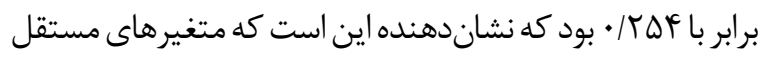

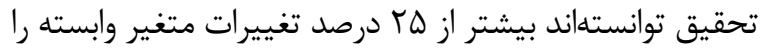

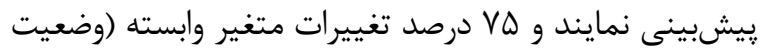

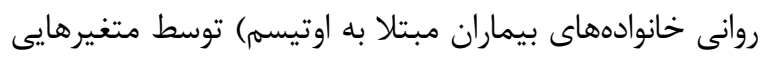

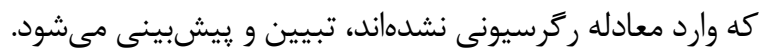

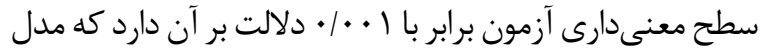
ركرسيونى تحقيق مدل مناسبى براى تبيين متغير وابسته است داست

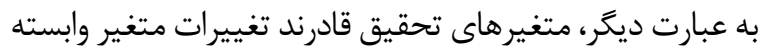
را توضيح دهند. نتايج تحقيق كنونى با مطالعه عالى و همكاران

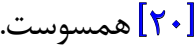

\section{$\sqrt{5}$}

اين يزوهش داراى محدوديتهايى بود مانند كمبود منابع فارسى در خصوص موضوع تحقيق، عدم همكارى بعضى از

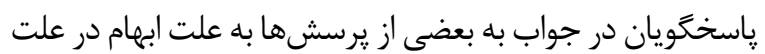

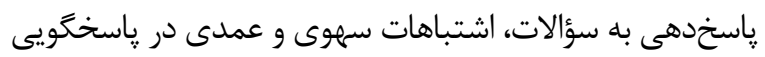

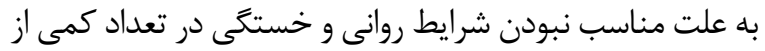

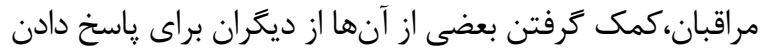

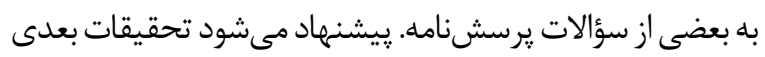

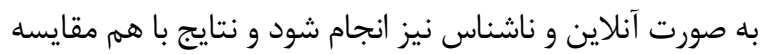

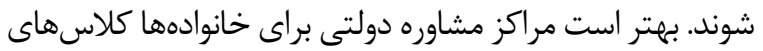


مىشود در تحقيقات بعدى در مورد جزئيات فضاى داخلى منازل

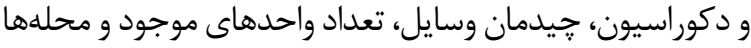

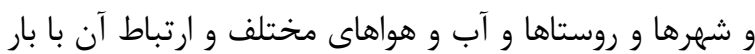

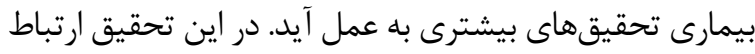

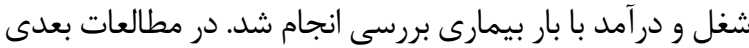

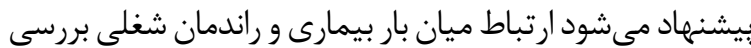

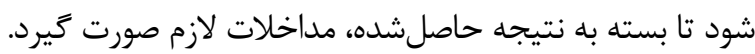

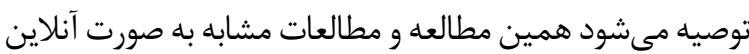

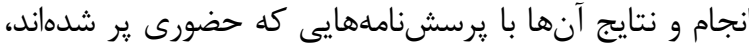

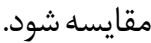

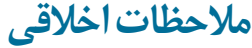

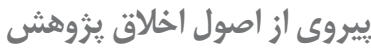

اصول اخلاقى تماماً در اين مقاله رعايت شده است. شركت

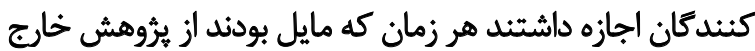

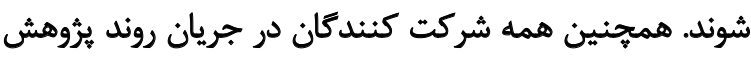
بودند. اطلاعات آن ها محرمانه نكه داشته شد شدر

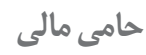

اين تحقيق هيجَّونه كمك مالى از سازمانهاى تأمين مالى در

بخشهاى عمومى، تجارى يا غير انتفاعى دريافت نكرد.

$$
\text { مشار كت نويسندكان }
$$

تمام نويسندكان در طراحى، اجراو نكارش همه بخشهاى ئروهش حاضر مشاركت داشتهاند.

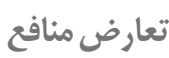

بنابر اظهار نويسندكان اين مقاله تعارض منافع ندارد.

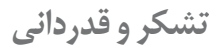

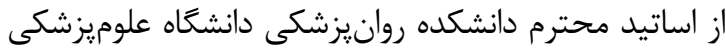

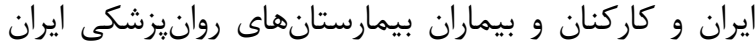

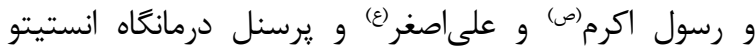

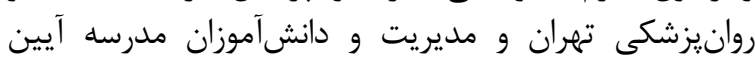
مهرورزى نهايت تشكر و قدردانى را داريم. 


\section{References}

[1] Karimi S, Javadi M, Jafarzadeh F. [Economic burden and costs of chronic diseases in iran and the world (Persian)]. Health Information Management. 2012; 8(7):984-96. http://him.mui.ac.ir/index.php/ $\mathrm{him} /$ article/view/522

[2] Ghalehbandi MF. [Textbook of Clinical psychiatry \& behavioral sciences (Persian)]. Tehran: Arjmand; 2018. http://opac.nlai.ir/opacprod/bibliographic/4921250

[3] Gandal MJ, Haney JR, Parikshak NN, Leppa V, Ramaswami G, Hartl $\mathrm{C}$, et al. Shared molecular neuropathology across major psychiatric disorders paralles polygenic overlap. Science. 2018; 359(6376):693-7. [DOI:10.1126/science.aad6469] [PMID] [PMCID]

[4] Masoumi T, Shafaroodi N, Razvan Z. Participation of people with Schizophrenia in everyday life. Iranian Rehabilitation Journal. 2018; 16(3):297-306. [DOI:10.32598/irj.16.3.297]

[5] Martin EA. Concise medical dictionary. Oxford: OUP Oxford; 2010. https://www.google.com/books/edition/Concise_Medical_Dictionary $/$ Zs8ZM4OUurcC?hl=en\&gbpv $=0$

[6] Large M, Sharma S, Compton MT, Slade T, Nielssen O. Cannabis use and earlier onset of psychosis: A systematic meta-analysis. Archives of General Psychiatry. 2011; 68(6):555-61. [DOI:10.1001/ archgenpsychiatry.2011.5] [PMID]

[7] Kaplan HI, Sadock's BJ. Kaplan and Sadock's synopsis of psychiatry: Behavioral sciences/clinical psychiatry. Philadelphia: Wolters Kluwer Health; 2016. [DOI:10.1097/00004850-198907000-00008]

[8] Martínez-Arán A, Vieta E, Reinares M, Colom F, Torrent C, SánchezMoreno J, et al. Cognitive function across manic or hypomanic, depressed, and euthymic states in bipolar disorder. The American Journal of Psychiatry. 2004; 161(2):262-70. [DOI:10.1176/appi. ajp.161.2.262] [PMID]

[9] Tamizi Z, Ranjbar F, Yaghmaei F, Alavi-Majd H, Fallahi-Khoshknab M. [Assessment of relationship between quality of life and coping strategies in schizophrenic patients in refer to psychiatric clinics of educational hospitals of Tehran (Persian)]. Archive of Rehabilitation. 2013; 13(4):124-31. http://rehabilitationj.uswr.ac.ir/article1-1271-en.html

[10] Nik-Farjam M, Forouzandeh N, Parvin N. [Dental health status of schizophrenic patients in the chronic psychiatric care center in the province of chaharmahal va bakhtivary (Persian)]. Archive of Rehabilitation. 2011;12(2):14-9. http://rehabilitationj.uswr.ac.ir/article1-343-en.html

[11] Fajutrao L, Locklear J, Priaulx J, Heyes A. A systematic review of the evidence of the burden of bipolar disorder in Europe. Clinical Practice and Epidemiology in Mental Health. 2009; 5:3. [DOI:10.1186/1745-0179-5-3] [PMID] [PMCID]

[12] Merikangas KR, Akiskal HS, Angst J, Greenberg PE, Hirschfeld RM, Petukhova M, et al. Lifetime and 12-month prevalence of bipolar spectrum disorder in the National Comorbidity Survey replication. Archives of General Psychiatry. 2007; 64(5):543-52. [DOI:10.1001/ archpsyc.64.5.543] [PMID] [PMCID]

[13] Sadock BJ, Kaplan HI, Sadock V. Kaplan and Sadock's Synopsis of Psychiatry. 10th ed. Philadelphia: Lippincott Williams \& Wilkins Co; 2007. https://www.google.com/books/edition/Kaplan_Sadock_s_ Synopsis_of_Psychiatry/u-ohbTtxCeYC?hl=en\&gbpv $=0$
[14] Dore G, Romans SE. Impact of bipolar affective disorder on family and partners. Journal of Affective Disorders. 2001; 67(1-3):147-58. [DOI:10.1016/S0165-0327(01)00450-5]

[15] Melo-Dias C. Psychosocial rehabilitation the Community ReEntry Program (CREP) in psychiatric inpatient settings, systematic review. American Journal of Nursing Science. 2015; 4(1-2):51-9. [DOI:10.11648/j.ajns.s.2015040201.20]

[16] Khodaei-Ardakani M R, Kamran-Derakhshan M, Rezaei O, Dolatshahi B, Mirab-Zadeh A. [Compare of executive function in Bipolar I Disorder and Schizophrenia (Persian)]. Archives of Rehabilitation. 2013; 14(3):73-9. http://rehabilitationj.uswr.ac.ir/article1-668-en.html

[17] Masi A, DeMayo MM, Glozier N, Guastella AJ. An overview of autism spectrum disorder, heterogeneity and treatment Options. Neuroscience Bulletin. 2017; 33(2):183-93. [DOI:10.1007/s12264017-0100-y] [PMID] [PMID]

[18] Sadigh Arfaee F, Dara N. [The effectiveness of quality of life psychotherapy on the psychological well-being of mothers of children with Autism (Persian)]. Qurterly Journal of Social Work. 2018; 6(4):23-32. http://socialworkmag.ir/article-1-204-fa.html

[19] Hoshiar M, Kakavand A, Ahmadi A. [The effectineness of mindfulness based therapy on quality of life and coping strategies of mothers of children with autistic spectrum disorder (Persian)]. Research Journal on Social Work. 2018; 7(2):32-9. https://www.sid.ir/en/journal/ ViewPaper.aspx?id=703319

[20] Aali S, AminYazdi SA, Abdekhodaei MS, Chamanabad AG, Moharreri F. [Designing family-centered combined treatment based on the human integrated development model andcomparing its effectiveness with floor time play therapy on behavioral symptoms of childrenwith autism spectrom disorder and the paranting stressof their mothers (Persian)]. Journal of Psychological Achivements. 2015; 22(1):25-46. [DOI:10.22055/PSY.2015.11179]

[21] Barekatain M, Attari A, Noori F, Aminoroaia M. [Assessment of psycho-educationil needs of families of patients with bipolar (Persian)]. Journal of Research in Behavioural Sciences. 2016; 14(1):23-9. [DOI:20.1001.1.17352029.1395.14.1.5.7]

[22] Xiang M, Ran M, Li S. A controlled evaluation of psychoeducational family intervention in a rural Chinese community. The British Journal of Psychiatry. 1994; 165(4):544-8. [DOI:10.1192/bjp.165.4.544] [PMID]

[23] Hudson P, Quinn K, Kristjanson L, Thomas T, Braithwaite M. Fisher J, et al. Evaluation of a psycho-educational group programme for family caregivers in home-based palliative care. Palliative Medicine. 2008; 22(3):270-80. [DOI:10.1177/0269216307088187] [PMID]

[24] Kristjanson LJ, Aoun S. Palliative care for families: Remembering the hidden patients. Canadian Journal of Psychiatry. 2004; 49(6):35965. [DOI:10.1177/070674370404900604] [PMID]

[25] Matson JL, Hattier MA, Belva B. Treating adaptive living skills of persons with autism using applied behavior analysis: A review. Research in Autism Spectrum Disorders. 2015; 6(1):271-6. [DOI:10.1016/j.rasd.2011.05.008]

[26] Ivey JK. What do parents expect? A study of likelihood and importance issues for children with autism spectrum disorders. Focus on Autism and Other Developmental Disabilities. 2014; 19(1):27-33. [D OI:10.1177/10883576040190010401] 
[27] Falloon IRH. Family interventions for mental disorders: Efficacy and effectiveness. World Psychiatry. 2003; 2(1):20-8. [PMID] [PMCID]

[28] Lane P, McKenna H, Ryan A, Fleming P. The experience of the family caregivers' role: A qualitative study. Research and Theory for Nursing Practice. 2003; 17(2):137-51. [DOI:10.1891/ rtnp.17.2.137.53173] [PMID]

[29] Geriani D, Savithry KSB, Shivakumar S, Kanchan T. Burden of care on caregivers of Schizophrenia patients: A correlation to personality and coping. Journal of Clinical and Diagnostic Research. 2015; 9(3):VC01-4. [DOI:10.7860/JCDR/2015/11342.5654] [PMID] [PMCID]

[30] Abdollahpour I, Nedjat S, Salimi Y, Noroozian M, Majdzadeh $R$. Which variable is the strongest adjusted predictor of quality of life in caregivers of patients with dementia? Psychogeriatrics. 2015; 15(1):51-7. [DOI:10.1111/psyg.12094] [PMID]

[31] Harvey K, Catty J, Langman A, Winfield H, Clement S, Burns $\mathrm{E}$, et al. A review of instruments developed to measure outcomes for carers of people with mental health problems. Acta Psychiatrica Scandinavica. 2008; 117(3):164-76 [DOI:10.1111/j.16000447.2007.01148.x] [PMID]

[32] Leucht S, Tardy M, Komossa K, Heres S, Kissling W, Salanti $\mathrm{G}$, et al. Antipsychotic drugs versus placebo for relapse prevention in schizophrenia: A systematic review and meta-analysis. The Lancet. 2012; 379(9831):2063-71. [DOI:10.1016/S01406736(12)60239-6] [PMID]

[33] Navidian A, Pahlavanzadeh S, Yazdani M. [The effectiveness of family training on family caregivers of inpatients with mental disorders (Persian)]. Iranian Journal of Psychiatry and Clinical Psychology. 2010; 16(2):99-106. http://ijpcp.iums.ac.ir/article1-1041-en.html

[34] Shamsaei F, Mohammadkhan Kermanshahi S, Vanaki Z. [Survey of family caregiver needs of patients with bipolar disorder (Persian)]. Avicenna Journal of Clinical Medicine. 2010; 17(3):57-63. http://sjh.umsha.ac.ir/article-1-268-en.html

[35] Haresabadi M, Bibak B, Hosein Zadeh E, Bayati M, Arki M, Akbari H. [Assessing burden of family caregivers of patients with schizophrenia Admitted in Imam Reza hospital- Bojnurd 2010 (Persian)]. Journal of North Khorasan University of Medical Science. 2012; 4(2):165-71. [DOI:10.29252/jnkums.4.2.165]

[36] Sadeghi M, Kaviani H, Rezai R. [Stigma of mental disorder among families of patients with major depressive disorder, bipolar disorder and Schizophrenia (Persian)]. Advances in Cognitive Sciences. 2003; 5(2):16-25. http://icssjournal.ir/article-1-163-en. html 
This Page Intentionally Left Blank 